\title{
ON A CLASS OF FULLY NONLINEAR FLOWS IN KÄHLER GEOMETRY
}

\author{
HAO FANG, MIJIA LAI, AND XINAN MA
}

\begin{abstract}
In this paper, we study a class of fully nonlinear metric flow on Kähler manifolds, which includes the J-flow as a special case. We provide a sufficient and necessary condition for the long time convergence of the flow, generalizing the result of SongWeinkove. As a consequence, under the given condition, we solved the corresponding Euler equation, which is fully nonlinear of Monge-Ampère type. As an application, we also discuss a complex Monge-Ampère type equation including terms of mixed degrees, which was first posed by Chen.
\end{abstract}

\section{INTRODUCTION}

In the study of Kähler geometry, the geometric flow method has been applied extensively to obtain "optimal" metrics. One classical example is the Kähler-Ricci flow. If the manifold has negative or vanishing first Chern class, the Kähler-Ricci flow converges to the Einstein metric, see Cao [C]. Another example is the so-called J-flow. It was introduced by Donaldson [D] in the setting of moment maps and by Chen in [Ch1, Ch2], as the gradient flow of the $J$-functional, which appears as a term of the Mabuchi energy. In [W1], Weinkove settled the question of Donaldson for surfaces. A sufficient class condition for the convergence of the J-flow is derived in [W2]. In [SW], Song and Weinkove proved a positivity condition to be equivalent to the convergence of the J-flow to a critical metric; The precise statement of this condition can be found in the discussion after (1.6). In general, the solution of these geometric flows usually depends on establishing a priori estimates of parabolic PDEs.

In this paper, we will study a class of fully non-linear geometric flows, which was motivated by the construction of J-flow.

Let $(M, \omega)$ be a closed Kähler manifold of dimension $n$. Define

$$
\mathcal{H}^{+}=\left\{[\chi] \in H^{1,1}(M), \quad \exists \chi \in[\chi], \chi>0\right\} .
$$

Let $[\chi] \in \mathcal{H}^{+}$and $\chi_{0} \in[\chi]$ is another Kähler form on $M$. We define the corresponding Kähler cone and Kähler potential space with respect to $[\chi]$ as

$$
\mathcal{K}_{[\chi]}=\left\{\chi_{\varphi}=\chi_{0}+\frac{\sqrt{-1}}{2} \partial \bar{\partial} \varphi>0, \quad \varphi \in C^{\infty}(M)\right\}
$$

The work of the first-named author is partially supported by NSF grant number DMS 060672. The work of the third-named author is partially supported by NSFC grant number 10671186, NSF grant number DMS 0635607 and Zürich Financial Services. 


$$
\mathcal{P}_{\chi_{0}}=\left\{\varphi \in C^{\infty}(M) \mid \chi_{\varphi}=\chi_{0}+\frac{\sqrt{-1}}{2} \partial \bar{\partial} \varphi>0\right\} .
$$

For a fixed integer $k \in[1, n]$, and $\lambda=\left(\lambda_{1}, \cdots, \lambda_{n}\right) \in \mathbb{R}^{n}$, the $k$-th elementary symmetric polynomial of $\lambda$ is defined as

$$
\begin{aligned}
\sigma_{0}(\lambda) & =\quad 1 ; \\
\sigma_{k}(\lambda) & =\sum_{1 \leq i_{1}<i_{2}<\cdots<i_{k} \leq n} \lambda_{i_{1}} \lambda_{i_{2}} \cdots \lambda_{i_{k}}, \quad k \geq 1 .
\end{aligned}
$$

When no confusion arises, we also use $\sigma_{k}(A)$ to denote the $k$-th elementary symmetric function of eigenvalues of a Hermitian matrix $A$.

In a local normal coordinate system of $M$ with respect to $\omega$, we have

$$
\chi_{0}=\frac{\sqrt{-1}}{2} \chi_{0 i \bar{j}} d z^{i} \wedge d z^{\bar{j}}, \quad \chi_{\varphi}=\frac{\sqrt{-1}}{2}\left(\chi_{0 i \bar{j}}+\varphi_{i \bar{j}}\right) d z^{i} \wedge d z^{\bar{j}} .
$$

Following the notation above, we denote

$$
\sigma_{k}\left(\chi_{\varphi}\right)=\left(\begin{array}{l}
n \\
k
\end{array}\right) \frac{\chi_{\varphi}^{k} \wedge \omega^{n-k}}{\omega^{n}}
$$

which is just the k-th elementary symmetric polynomial of the eigenvalues of the matrix $\left(\chi_{0 i} i+\varphi_{i \bar{j}}\right)$ with respect to the background metric $\omega$.

We set the volume form on $M$ as $d v=\omega^{n} / n$ !. It is clear that

$$
\begin{aligned}
c_{k} & =c_{k,[\omega],[\chi]}=\frac{\int_{M} \chi_{0}^{n-k} \wedge \omega^{k}}{\int_{M} \chi_{0}^{n}}, \\
c_{k}^{\prime} & =c_{k,[\omega],[\chi]}^{\prime}=\frac{\left(\begin{array}{l}
n \\
k
\end{array}\right) \int_{M} \chi_{0}^{n-k} \wedge \omega^{k}}{\int_{M} \chi_{0}^{n}}=\frac{\int_{M} \sigma_{n-k}\left(\chi_{0}\right) d v}{\int_{M} \sigma_{n}\left(\chi_{0}\right) d v}
\end{aligned}
$$

are topological constants. Now we consider following flow in $\mathcal{P}_{\chi_{0}}$ :

$$
\begin{aligned}
\chi_{t} & =\chi_{0}+\frac{\sqrt{-1}}{2} \partial \bar{\partial} \varphi_{t}, \\
\frac{\partial \varphi_{t}}{\partial t} & =c_{k}^{\prime \frac{1}{k}}-\left(\frac{\sigma_{n-k}\left(\chi_{\varphi_{t}}\right)}{\sigma_{n}\left(\chi_{\varphi_{t}}\right)}\right)^{\frac{1}{k}} \\
\varphi_{0} & =0 .
\end{aligned}
$$

Clearly, the stationary metric of this flow is a Kähler metric $\chi \in \mathcal{K}_{[\chi]}$ satisfying:

$$
\chi^{n-k} \wedge \omega^{k}=c_{k} \chi^{n}=\left(\frac{\int \chi_{0}^{n-k} \wedge \omega^{k}}{\int \chi_{0}^{n}}\right) \chi^{n} .
$$

In the case of $k=1$, our flow is same as the $J$-flow. Song-Weinkove [SW] gave a sufficient and necessary condition for the J-flow to exist and converge to a solution of (1.5).

One of the purposes of this paper is to give a necessary and sufficient condition for the flow (1.4) to converge to the stationary metric, which we now describe as a cone condition. For $M$ and $\omega$ given as above, we define $\mathcal{C}_{k}=\mathcal{C}_{k}(\omega)$ as

$$
\mathcal{C}_{k}(\omega)=\left\{[\chi] \in \mathcal{H}^{+}, \exists \chi^{\prime} \in[\chi] \text {, s.t. } c_{k} n \chi^{\prime n-1}>(n-k) \chi^{\prime n-k-1} \wedge \omega^{k}\right\} .
$$


$\mathcal{C}_{k}$ is an affine cone in $\mathcal{H}^{+}$. For $k=1, C_{1}$ is first defined in [SW]. It is easy to check that $[\chi] \in \mathcal{C}_{k}$ is a necessary condition for the equation (1.5) to be solvable (see Section 2 for more details). The main theorem of this paper is the following

Theorem 1.1. Suppose $M, \omega$ and $\chi_{0} \in[\chi]$ are defined as above. Let $1 \leq k \leq n$. If $[\chi] \in \mathcal{C}_{k}(\omega)$, then flow 1.4) has a long time solution, which converges to a smooth metric satisfying (1.5).

It is worthwhile to point out the case of $k=n$. Notice that the corresponding equation is equivalent to

$$
\chi_{\varphi}^{n}=\frac{\int_{M} \chi_{0}^{n}}{\int_{M} \Omega} \Omega
$$

where $\Omega$ is any given volume form. This was solved by Yau in his celebrated paper $[\mathrm{Y}]$. Also notice that the condition (1.6) becomes trivial in this case; in other words, $\mathcal{C}_{n}=\mathcal{H}^{+}$. Cao [C] provides a parabolic approach to this equation, using Ricci flow.

Notice that for the $k=1$ case, our condition and conclusion are exactly same as the ones in $[\mathrm{SW}$.

Theorem 1.1 can be viewed as a finite interpolation between results of Yau [Y], Cao [C], Song-Weinkove [SW]. In fact, our basic approach to prove Threorem 1.1 closely follows these earlier works. In particular, the idea of establishing partial $C_{0}$ estimate before $C_{2}$ and $C_{0}$ estimates first appears in [W1. However, new convexity phoneomena shows up for $k \neq 1, n$ cases.

Theorem 1.1 can be understood from several aspects.

First, Theorem 1.1 can be understood geometrically. One motivation for the construction of this flow (1.4), as well as an important ingredient of the proof of Theorem 1.1, is the following functional defined for $\chi_{\phi}$ with $\phi \in \mathcal{P}_{\chi_{0}}$ and $j \geq 0$,

$$
\mathcal{F}_{j}\left(\chi_{\phi}\right)=\int_{0}^{1} \int_{M} \frac{\partial \phi_{t}}{\partial t} \chi_{\phi_{t}}^{j} \wedge \omega^{n-j} d t
$$

where $\phi_{t} \in \mathcal{P}_{\chi_{0}}, t \in[0,1]$ is a path in connecting $\chi_{0}$ and $\chi_{\phi} . \mathcal{F}_{j}$ is shown to be independent of the choice of path [ChT. Furthermore, a functional defined as

$$
\tilde{\mathcal{F}}_{j, n}\left(\chi_{0}, \chi_{\phi}\right)=\mathcal{F}_{j}\left(\chi_{\phi}\right)-c_{n-j} \mathcal{F}_{n}\left(\chi_{\phi}\right)
$$

can be viewed as a functional depending only on $\chi_{0}, \chi_{\phi} \in \mathcal{K}_{\chi}$.

Notice that for $\chi_{i} \in[\chi], i=0,1,2$, we have

$$
\tilde{\mathcal{F}}_{j, n}\left(\chi_{0}, \chi_{1}\right)+\tilde{\mathcal{F}}_{j, n}\left(\chi_{1}, \chi_{2}\right)=\tilde{\mathcal{F}}_{j, n}\left(\chi_{0}, \chi_{2}\right) .
$$

Thus, the minimizer of functional $\tilde{\mathcal{F}}_{j, n}\left(\chi_{0}, \cdot\right)$ is independent of the choice $\chi_{0}$. In fact, this functional can be realized as quotients of Quillen metrics on the determinant bundles with certain virtual bundle coefficients, see Tian [T2].

Our flow (1.4) is constructed in such a way that the functional $\tilde{\mathcal{F}}_{n-k, n}\left(\chi_{0}, \chi_{\varphi_{t}}\right)$ is decreasing along the flow. It is then easy to check that the corresponding minimum metric 
satisfies (1.5). Theorem 1.1 gives an explicit path for the functional $\tilde{\mathcal{F}}_{n-k, n}\left(\chi_{0}, \chi\right)$ to obtain its unique minimal, when the cone condition $[\chi] \in \mathcal{C}_{k}$ is satisfied. Notice that our flow is not the gradient flow of the corresponding functionals except the case $k=1$. In fact, we modified the functional's gradient flow to ensure certain PDE estimates hold.

Second, Theorem 1.1 provides a necessary and sufficient condition for (1.5), an elliptic equation of Monge-Ampère type to be solvable. Notice that (1.5) can be written, locally, for $k<n$ as

$$
c_{k}^{\prime} \sigma_{n}\left(\chi_{\varphi}\right)=\sigma_{n-k}\left(\chi_{\varphi}\right),
$$

or, equivalently,

$$
\sigma_{k}\left(\chi_{\varphi}^{-1}\right)=c_{k}^{\prime} .
$$

The corresponding $[\chi] \in \mathcal{C}_{k}$ condition states that there exists a $\chi^{\prime} \in[\chi]$ such that

$$
\sigma_{k}\left(\chi^{\prime-1} \mid i\right)<c_{k}^{\prime}
$$

for $1 \leq i \leq n$. Refer to Section 2 for more details.

Equation (1.5) is also a special case of a question posed by Chen. In [Ch1, Chen raised the question of solving a very general fully non-linear equation of Monge-Ampère type:

$$
\chi_{\varphi}^{n}=\sum_{i=0}^{n-1} \alpha_{i} \chi_{\varphi}^{i} \wedge \omega^{n-i},
$$

where $\alpha_{i}$ 's are real. Theorem 1.1 gives a complete answer for Chen's question when the right hand side has only one term.

Using similar method, we can also extend our result.

Define, for any fixed $\alpha \in(0, \infty)$ and integer $k \in[1, n]$,

$$
\begin{aligned}
c_{k, \alpha}= & c_{k, \alpha,[\omega],[\chi]}=c_{k}+\alpha c_{k-1}, \\
\tilde{\mathcal{F}}_{\alpha, k, n}\left(\chi_{0}, \chi\right)= & \tilde{\mathcal{F}}_{n-k, n}\left(\chi_{0}, \chi\right)+\alpha \tilde{\mathcal{F}}_{n-k+1, n}\left(\chi_{0}, \chi\right), \\
\mathcal{C}_{k, \alpha}(\omega)= & \left\{[\chi] \in \mathcal{H}^{+}, \exists \chi^{\prime} \in[\chi],\right. \text { such that } \\
& \left.c_{k, \alpha} n \chi^{\prime n-1}>(n-k) \chi^{\prime n-k-1} \wedge \omega^{k}+\alpha(n-k+1) \chi^{\prime n-k} \wedge \omega^{k-1}\right\} .
\end{aligned}
$$

It is clear to see that when the parameter $\alpha$ runs from 0 to $\infty, \mathcal{C}_{k, \alpha}=\mathcal{C}_{k, \alpha}(\omega)$ gives a continuous deformation from the cone $\mathcal{C}_{k} \subset \mathcal{H}^{+}$to $\mathcal{C}_{k-1} \subset \mathcal{H}^{+}$. We have the following

Theorem 1.2. Suppose $M, \omega$ and $\chi_{0} \in[\chi]$ are defined as above. Assume $1 \leq k \leq n$ and $\alpha>0$, then the equation

$$
c_{k, \alpha} \chi^{n}=\chi^{n-k} \wedge \omega^{k}+\alpha \chi^{n-k+1} \wedge \omega^{k-1}
$$

has a unique smooth solution if and only if $[\chi] \in \mathcal{C}_{k, \alpha}(\omega)$; in this case, the solution minimizes $\tilde{\mathcal{F}}_{\alpha, k, n}\left(\chi_{0}, \chi\right)$.

Theorem 1.2 is proved by improving the estimates needed in proving Theorem 1.1 to the product manifold $M \times C$, where $C$ is a smooth algebraic curve.

Based on these known results, we would like to verify that the similar cone condition would be the necessary and sufficient condition for the problem of Chen. Using a similar 
geometric construction as in the proof of Theorem 1.2, we can settle many special cases for Chen's problem. See Section 5 for more details. We believe this is one of the few examples of the Monge-Ampère type equations including terms of mixed degrees. The geometric structure plays an important role in the solution of these equations.

Finally, we make some remarks.

Remark 1.3. It is interesting to point out that the elliptic PDEs studied in this paper are all solved by geometric flow method. With the exception of Yau's original equation, continuity method does not seem to work for the other cases.

Remark 1.4. It is interesting to study the various cones we defined in $\mathcal{H}^{+}$. Except the obvious fact that $\mathcal{C}_{n}(\omega)=\mathcal{H}^{+}$includes all the other cones, the relative position of $\mathcal{C}_{j}(\omega)$ and $\mathcal{C}_{k}(\omega)$ for $j \neq k, j, k \neq n$ is unknown.

Remark 1.5. The strong concavity property of the symmetric polynomials is very important for our estimates. We point out that we do not use the optimal concavity property available. This leaves room of future construction of other geometric flows in Kähler geometry.

The rest of this paper is organized as follows. In Section 2 we introduce further notation and some preliminary facts about the elementary symmetric polynomials. In Section 3, we derive the partial $C^{2}$ estimate by maximum principle, following Yau [Y] and Weinkove [W1]. In Section 4, we derive the $C^{0}$ estimate and $C^{\infty}$ estimate and the convergence result. In section 5 , we discuss various generalization of Theorem 1.1 and some application to complex geometry. In the Appendix, we give an alternative proof of our strong concavity property.

Acknowledgments: The first-named author would like to thank Jian Song for useful discussion. All authors would like to thank Pengfei Guan and Lihe Wang for discussion. They would like to thank Institute for Advanced Study for support and hospitality. Most of this work is done when they attended special year of Geometric non-linear PDE at IAS. Thanks also go to referee for his or her careful proof-reading and useful suggestion.

\section{Preliminary}

In this section, we set up the notation and prove some preliminary results regarding elementary symmetric functions.

For simplicity, after proper scaling, we may assume $c_{k}=\frac{\int \chi_{0}^{n-k} \wedge \omega^{k}}{\int \chi_{0}^{n}}=1$ without loss of generality. We also denote $c=c_{k}^{\prime}=\left(\begin{array}{l}n \\ k\end{array}\right)$ when no confusion occurs. 
Fix a local coordinate chart $U \subset M$. For $z=\left(z_{1}, z_{2}, \cdots, z_{n}\right) \in U$, we write

$$
\begin{aligned}
\omega & =\frac{\sqrt{-1}}{2} g_{i \bar{j}} d z^{i} \wedge d z^{\bar{j}}, \\
\chi_{0} & =\frac{\sqrt{-1}}{2} \chi_{0 i \bar{j}} d z^{i} \wedge d z^{\bar{j}}, \\
\chi^{\prime} & =\frac{\sqrt{-1}}{2} \chi_{i \bar{j}}^{\prime} d z^{i} \wedge d z^{\bar{j}}, \\
\chi_{\varphi} & =\frac{\sqrt{-1}}{2}\left(\chi_{0 i \bar{j}}+\varphi_{i \bar{j}}\right) d z^{i} \wedge d z^{\bar{j}}, \\
\chi_{i \bar{j}} & =\chi_{0 i \bar{j}}+\varphi_{i \bar{j}} .
\end{aligned}
$$

When no confusion occurs, we also use $\chi_{0}, \chi^{\prime}, \chi_{\varphi}$ to denote the corresponding Hermitian matrices at the given $z$. We always choose the normal coordinate of $\omega$ such that $g_{i \bar{j}}=\delta_{i \bar{j}}$ and $\chi_{\varphi}$ is diagonal. In other words, we have $\chi_{\varphi}(z)=\chi=\left(\chi_{1}, \cdots, \chi_{n}\right)$. Furthermore, we may assume $\chi_{i} \geq \chi_{j}$ for $i>j$. That means $\chi_{1}$ and $\chi_{n}$ are the maximal and the minimal eigenvalues of $\chi_{\varphi}$, respectively.

For a Hermitian matrix $A=\left(a_{i \bar{j}}\right)_{n \times n}$, define

$$
F(A):=-\left[\frac{\sigma_{n-k}(A)}{\sigma_{n}(A)}\right]^{\frac{1}{k}}=-\sigma_{k}^{\frac{1}{k}}\left(A^{-1}\right) .
$$

It is a well known fact that $\mathrm{F}$ is a concave function of $A$ and $F^{i \bar{j}}$ is positive definite when restricted to the space of positive definite hermitian matrixes (see e.g., $[\mathbf{S}$ ). Without further specification, we assume that $A$ is positive in the rest of this section.

We compute the derivatives of $F$ with respect to entries of $A$ for the future use.

Proposition 2.1. For $F$ given as above, we have

$$
\begin{gathered}
F^{i \bar{j}}(A):=\frac{\partial F}{\partial a_{i \bar{j}}}=-\frac{1}{k}\left(\frac{\sigma_{n-k}}{\sigma_{n}}\right)^{1 / k-1}\left(\frac{\partial \sigma_{n-k} / \partial a_{i \bar{j}}}{\sigma_{n}}-\frac{\sigma_{n-k} \partial \sigma_{n} / \partial a_{i \bar{j}}}{\sigma_{n}^{2}}\right) . \\
F^{i \bar{j}, k \bar{l}}(A):=\frac{\partial^{2} F(A)}{\partial a_{i \bar{j}} \partial a_{k \bar{l}}} .
\end{gathered}
$$

If $A=\chi=\operatorname{diag}\left(\chi_{1}, \chi_{2}, \cdots, \chi_{n}\right)$ is diagonal, then $F^{i \bar{j}}$ can be non trivial iff $i=j$. We have

or

$$
F^{i \bar{i}}=-\frac{1}{k}\left(\frac{\sigma_{n-k}(\chi)}{\sigma_{n}(\chi)}\right)^{1 / k-1}\left(\frac{\sigma_{n-k-1}(\chi \mid i)}{\sigma_{n}(\chi)}-\frac{\sigma_{n-k}(\chi) \sigma_{n-1}(\chi \mid i)}{\sigma_{n}^{2}(\chi)}\right)
$$

$$
F^{i \bar{i}}=\frac{1}{k} \sigma_{k}^{\frac{1}{k}-1}\left(\chi^{-1}\right) \sigma_{k-1}\left(\chi^{-1} \mid i\right) \frac{1}{\chi_{i}^{2}}
$$

Furthermore, $F^{i \bar{j}, k \bar{l}}$ can be nontrivial iff $i=j, k=l$ or $i=l, j=k$. In this case, we have

$$
F^{i \bar{j}, j \bar{i}}(\chi)=\frac{1}{k}\left(\frac{\sigma_{n-k}(\chi)}{\sigma_{n}(\chi)}\right)^{\frac{1}{k}-1}\left(\frac{\sigma_{n}(\chi) \sigma_{n-k-2}(\chi \mid i, j)-\sigma_{n-k}(\chi) \sigma_{n-2}(\chi \mid i, j)}{\sigma_{n}^{2}(\chi)}\right), \text { for } i \neq j,
$$

where $\chi^{-1}$ denotes the inverse matrix of $\chi, \sigma_{k}(\chi \mid i)=\left.\sigma_{k}(\chi)\right|_{\chi_{i}=0}, \sigma_{k}(\chi \mid i, j)=\left.\sigma_{k}(\chi)\right|_{\chi_{i}=0, \chi_{j}=0}$. 
Also notice that $F$ is homogenous of degree -1 , so $-F(A)=\sum_{i, j} F^{i \bar{j}}(A) a_{i \bar{j}}$.

We proceed to discuss some technical results. First of all, we have the following concavity result. Define

$$
\Gamma_{n}=\left\{\left(x_{1}, \cdots, x_{n}\right) \in \mathbb{R}^{n} \mid x_{1}>0, x_{2}>0, \cdots x_{n}>0\right\} .
$$

Proposition 2.2. [GLZ] Let $g(\lambda)=\log \sigma_{k}(\lambda)$. For $\lambda \in \Gamma_{n}, \xi=\left(\xi_{1}, \cdots, \xi_{n}\right) \in \mathbb{C}^{n}$, let $g_{i}:=\frac{\partial g}{\partial \lambda_{i}}, g_{i j}:=\frac{\partial^{2} g}{\partial \lambda_{i} \partial \lambda_{j}}$, we have

$$
\sum_{i=1}^{n}\left(g_{i i}+\frac{g_{i}}{\lambda_{i}}\right) \xi_{i} \bar{\xi}_{i}+\sum_{i \neq j} g_{i j} \xi_{i} \bar{\xi}_{j} \geq 0
$$

Proof. We have

$$
g_{i}=\frac{\sigma_{k-1}(\lambda \mid i)}{\sigma_{k}(\lambda)}, \quad g_{i j}=\frac{\sigma_{k-2}(\lambda \mid i, j)}{\sigma_{k}(\lambda)}-\frac{\sigma_{k-1}(\lambda \mid i) \sigma_{k-1}(\lambda \mid j)}{\sigma_{k}^{2}(\lambda)} .
$$

Using the same reduction in Lemma 2.3 of [GM], (2.1) can be reduced to the following inequality

$$
\begin{array}{r}
\sum_{i=1}^{n} \sigma_{k}(\lambda \mid i) \sigma_{n-1}(\lambda \mid i) \sigma_{k-1}(\lambda \mid i)\left|\xi_{i}\right|^{2} \\
\geq \sigma_{n}(\lambda) \sum_{i \neq j}\left\{\sigma_{k-1}^{2}(\lambda \mid i j)-\sigma_{k}(\lambda \mid i j) \sigma_{k-2}(\lambda \mid i j)\right\} \xi_{i} \bar{\xi}_{j},
\end{array}
$$

which is just Lemma 2.4 in [GM].

Remark 2.3. By the above proposition, if we let $g(\lambda)=\sigma_{k}^{\frac{1}{k}}(\lambda)$, for $\lambda \in \Gamma_{n}$, then a simple calculation shows, for $\xi=\left(\xi_{1}, \cdots, \xi_{n}\right) \in \mathbb{C}^{n}$,

$$
\left(g_{i j}+\frac{g_{i}}{\lambda_{j}} \delta_{i j}\right) \xi_{i} \bar{\xi}_{j} \geq 0 .
$$

Another proof will be given in the appendix.

Second, we have the following local version of the cone condition (1.6).

Proposition 2.4. For $k<n, \chi^{\prime} \in \mathcal{C}_{k}$ is equivalent to

$$
\frac{\sigma_{n-k-1}\left(\chi^{\prime} \mid j\right)}{\sigma_{n-1}\left(\chi^{\prime} \mid j\right)}=\sigma_{k}\left(\chi^{\prime-1} \mid j\right)<\left(\begin{array}{l}
n \\
k
\end{array}\right),
$$

for any $j \in\{1, \cdots, n\}$, where $\left(\chi^{\prime} \mid j\right)$ denotes the matrix obtained by deleting the $j$-th column and $j$-th row of $\chi^{\prime}$.

Proof. Assume $\chi^{\prime} \in \mathcal{C}_{k}$. By (1.6) , for any given integer $j \in[1, n]$, the coefficient of the $(n-1, n-1)$ form $\Pi_{i=1 i \neq j}^{n} d z^{i} d \bar{z}^{i}$ in $\chi^{\prime n-1}-\frac{n-k}{n} \omega^{k} \wedge \chi^{\prime n-k-1}$ should be positive; that is,

$$
(n-1) ! \sigma_{n-1}\left(\chi^{\prime} \mid j\right)-\frac{n-k}{n} k !(n-k-1) ! \sigma_{n-k-1}\left(\chi^{\prime} \mid j\right)>0 .
$$


Dividing both sides by $\frac{n-k}{n} k !(n-k-1) ! \sigma_{n-1}\left(\chi^{\prime} \mid j\right)$, one obtains

$$
\frac{\sigma_{n-k-1}\left(\chi^{\prime} \mid j\right)}{\sigma_{n-1}\left(\chi^{\prime} \mid j\right)}<\left(\begin{array}{l}
n \\
k
\end{array}\right)
$$

Next, we introduce some simple algebraic facts. Let $A=\left(a_{i \bar{j}}\right)$ be a positive Hermitian matrix.

Lemma 2.5. Let $I=\left(i_{1}, i_{2}, \cdots, i_{k}\right) \subset(1, \cdots, n)$ be an index set, denote its complement in $(1,2, \cdots, n)$ by $\bar{I}$. We always order $\bar{I}$ so that $(I, \bar{I})$ is an even permutation of $(1,2, \cdots, n)$. For $A$, a positive hermitian $n \times n$ matrix, let $A_{I}$ be the principal minor $\left(a_{i j}\right)_{i, j \in I}$. Then

$$
\operatorname{det}(A) \leq \operatorname{det}\left(A_{I}\right) \operatorname{det}\left(A_{\bar{I}}\right)
$$

Proof. Rearrange $A$ if necessary we may write $A$ as

$$
A=\left[\begin{array}{cc}
A_{I} & M \\
M^{\prime} & A_{\bar{I}}
\end{array}\right]
$$

By

$$
\left[\begin{array}{cc}
I d & 0 \\
-M^{\prime} A_{I}^{-1} & I d
\end{array}\right]\left[\begin{array}{cc}
A_{I} & M \\
M^{\prime} & A_{\bar{I}}
\end{array}\right]=\left[\begin{array}{cc}
A_{I} & M \\
0 & A_{\bar{I}}-M^{\prime} A_{I}^{-1} M
\end{array}\right]
$$

one obtains

$$
\operatorname{det}(A)=\operatorname{det}\left(A_{I}\right) \operatorname{det}\left(A_{\bar{I}}-M^{\prime} A_{I}^{-1} M\right) \leq \operatorname{det}\left(A_{I}\right) \operatorname{det}\left(A_{\bar{I}}\right),
$$

where $M^{\prime}$ means the conjugate transpose matrix of $M$. The last inequality follows from the fact that $M^{\prime} A_{I}^{-1} M$ is positive definite.

The following corollary is a direct consequence of Lemma 2.5 .

Corollary 2.6. Let $A$ be as above. Then $\operatorname{det}(A) \leq \prod_{i=1}^{n} a_{i \bar{i}}$.

We are then ready to prove

Lemma 2.7. Let $A=\left(a_{i \bar{j}}\right)$ be a positive Hermitian matrix. Denote $\tilde{A}=\left(a_{i \bar{j}} \delta_{i \bar{j}}\right)$ to be the matrix containing only the diagonal terms of $A$. We have,

$$
\sigma_{k}\left(\tilde{A}^{-1}\right) \leq \sigma_{k}\left(A^{-1}\right)
$$

Proof. By Corollary 2.6, we have

$$
\frac{1}{\operatorname{det}(\tilde{A})} \leq \frac{1}{\operatorname{det}(A)}
$$


This means that Lemma 2.7 holds for $k=n$. For general $k$, we have

$$
\begin{aligned}
\sigma_{k}\left(\tilde{A}^{-1}\right) & =\sum_{|I|=k,\left(i_{1}, i_{2}, \cdots, i_{k}\right) \in I} \frac{1}{a_{i_{1} \overline{i_{1}}}} \frac{1}{a_{i_{2} \overline{i_{2}}}} \cdots \frac{1}{a_{i_{k} \overline{i_{k}}}} \\
& \leq \sum_{|I|=k} \frac{1}{\operatorname{det}\left(A_{I}\right)} \leq \sum_{|I|=k} \frac{\operatorname{det}\left(A_{\bar{I}}\right)}{\operatorname{det}(A)}=\frac{\sigma_{n-k}(A)}{\sigma_{n}(A)}=\sigma_{k}\left(A^{-1}\right) .
\end{aligned}
$$

Finally, we give the following technical statement, which will be used in the next section.

Theorem 2.8. Assume that $M, \omega, \chi \in[\chi]$ given as before. Assume that $k<n$ and $[\chi] \in \mathcal{C}_{k}$. Let $F^{i \bar{i}}(\chi)$ be given as in Proposition [2.1. Let $\chi^{\prime} \in[\chi]$ be the Kähler form satisfying the condition of $\mathcal{C}_{k}$. Assume $C_{1} \leq \frac{\sigma_{n-k}(\chi)}{\sigma_{n}(\chi)} \leq C_{2}$, for some universal constants $C_{1}$ and $C_{2}$. Then there exists a universal constant $N$, depending only on the given geometric data, such that, if $\frac{\chi_{1}}{\chi_{n}} \geq N$ then there exists $\epsilon>0$ such that

$$
(1-\epsilon) \sum_{i=1}^{n} F^{i \bar{i}}(\chi) \chi_{i \bar{i}}^{\prime} \geq c^{-\frac{1}{k}} \sigma_{k}^{\frac{2}{k}}\left(\chi^{-1}\right)
$$

Proof. Follow the convention, we will verify (2.6) under normal coordinates which diagonalizes $\chi$ at some point. So $\chi=\operatorname{diag}\left(\chi_{1}, \chi_{2}, \cdots \chi_{n}\right)$, and $\chi_{1} \geq \chi_{2} \geq \cdots \geq \chi_{n}$. In local coordinates we will use $\sigma_{k}\left(\chi^{-1}\right)=\frac{\sigma_{n-k}(\chi)}{\sigma_{n}(\chi)}$ when no confusion arises.

We first notice for the case $\chi_{n} \ll 1$, (2.6) follows easily. Notice $\chi^{\prime}$ is a fixed kähler form, so there is a constant $\lambda>0$ such that

$$
\chi^{\prime}>\lambda \omega
$$

Therefore,

$$
\begin{aligned}
\sum_{i=1}^{n} F^{i \bar{i}}(\chi) \chi_{i \bar{i}}^{\prime} & \geq \lambda \sum_{i=1}^{n} F^{i \bar{i}}(\chi) \\
& =\lambda \frac{1}{k} \sigma_{k}^{\frac{1}{k}-1}\left(\chi^{-1}\right) \sum_{i=1}^{n} \sigma_{k-1}\left(\chi^{-1} \mid i\right) \frac{1}{\chi_{i}^{2}} \\
& \geq \lambda \frac{1}{k} \sigma_{k}^{\frac{1}{k}-1}\left(\chi^{-1}\right) \sigma_{k-1}\left(\chi^{-1} \mid n\right) \frac{1}{\chi_{n}^{2}}
\end{aligned}
$$

We claim $\sigma_{k-1}\left(\chi^{-1} \mid n\right) \frac{1}{\chi_{n}}$ is bounded below. Indeed, $\sigma_{k-1}\left(\chi^{-1} \mid n\right) \frac{1}{\chi_{n}}$ is the largest term among $\sigma_{k-1}\left(\chi^{-1} \mid i\right) \frac{1}{\chi_{i}}$ by the fact that $\chi_{n}$ is the smallest among $\chi_{i}, 1 \leq i \leq n$. Thus,

$$
\sigma_{k-1}\left(\chi^{-1} \mid n\right) \frac{1}{\chi_{n}} \geq 1 / n\left[\sum_{i=1}^{n} \sigma_{k-1}\left(\chi^{-1} \mid i\right) \frac{1}{\chi_{i}}\right]=\frac{k}{n} \sigma_{k}\left(\chi^{-1}\right)
$$

Now if $\chi_{n}<\delta=\lambda\left(C_{1} c\right)^{\frac{1}{k}}$, (2.6) follows easily from (2.7) and (2.8).

So we just need to consider the case $\chi_{n} \geq \delta$. 
Recall Gårding's inequality: For $\mu, \tau \in \Gamma_{n}$,

$$
\frac{1}{k} \sum_{j=1}^{n} \tau_{j} \frac{\partial}{\partial \mu_{j}} \sigma_{k}(\mu) \geq \sigma_{k}^{\frac{1}{k}}(\tau) \sigma_{k}^{1-\frac{1}{k}}(\mu) .
$$

Thus, by Proposition 2.1, we have, for the matrix $B=\operatorname{diag}\left(\frac{\chi_{1 \overline{1}}^{\prime}}{\chi_{1}^{2}}, \cdots, \frac{\chi_{n \bar{n}}^{\prime}}{\chi_{n}^{2}}\right)=\chi^{-1} \tilde{\chi}^{\prime} \chi^{-1}$,

$$
\begin{aligned}
\sum_{i=1}^{n} F^{i \bar{i}}(\chi) \chi_{i \bar{i}}^{\prime} & =\sigma_{k}^{\frac{1}{k}-1}\left(\chi^{-1}\right) \frac{1}{k} \sum_{i=1}^{n} \sigma_{k-1}\left(\chi^{-1} \mid i\right) \frac{\chi_{i \bar{i}}^{\prime}}{\left(\chi_{i}\right)^{2}} \\
& \geq \sigma_{k}^{\frac{1}{k}-1}\left(\chi^{-1}\right) \sigma_{k}^{1-\frac{1}{k}}\left(\chi^{-1}\right) \sigma_{k}^{\frac{1}{k}}(B) \\
& =\sigma_{k}^{\frac{1}{k}}(B) .
\end{aligned}
$$

Comparing with (2.6), it suffices to show

$$
c^{\frac{1}{k}} \sigma_{k}^{\frac{1}{k}}(B) \geq(1+\theta) \sigma_{k}^{\frac{2}{k}}\left(\chi^{-1}\right), \text { for } \quad \theta>0 .
$$

By Proposition 2.4, we have

$$
\sigma_{k}\left(\left(\chi^{\prime} \mid 1\right)^{-1}\right) \leq\left(\begin{array}{l}
n \\
k
\end{array}\right)-\eta=c-\eta
$$

for a universal positive constant $\eta<c$, depending only on $(M, \omega)$ and $\chi^{\prime}$, where $\left(\chi^{\prime} \mid 1\right)^{-1}$ is the inverse matrix of $\left(\chi^{\prime} \mid 1\right)$. We have,

$$
\begin{aligned}
c^{\frac{1}{k}} \sigma_{k}^{\frac{1}{k}}(B) & \geq\left(\frac{c}{c-\eta}\right)^{\frac{1}{k}} \sigma_{k}^{\frac{1}{k}}\left(\left(\chi^{\prime} \mid 1\right)^{-1}\right) \sigma_{k}^{\frac{1}{k}}(B) \\
& \geq\left(\frac{c}{c-\eta}\right)^{\frac{1}{k}} \sigma_{k}^{\frac{1}{k}}\left(\left(\chi^{\prime} \mid 1\right)^{-1}\right) \sigma_{k}^{\frac{1}{k}}(B) \\
& \geq\left(\frac{c}{c-\eta}\right)^{\frac{1}{k}} \sigma_{k}^{\frac{1}{k}}\left(\left(\chi^{\prime} \mid 1\right)^{-1}\right) \sigma_{k}^{\frac{1}{k}}(B \mid 1) \\
& \geq\left(\frac{c}{c-\eta}\right)^{\frac{1}{k}} \sigma_{k}^{\frac{2}{k}}\left(\chi^{-1} \mid 1\right) .
\end{aligned}
$$

We explain the second and last inequality in (2.12). Apply Lemma 2.7 to the matrix $\left(\chi^{\prime} \mid 1\right)$, we have

$$
\sigma_{k}\left(\left(\chi^{\prime} \mid 1\right)^{-1}\right) \geq \sigma_{k}\left(\left(\chi^{\prime} \mid 1\right)^{-1}\right)
$$

Recall that $B=\chi^{-1} \tilde{\chi}^{\prime} \chi^{-1}$, then Cauchy-Schwarz inequality yields

$$
\sigma_{k}\left(\chi^{-1} \tilde{\chi}^{\prime} \chi^{-1} \mid 1\right) \sigma_{k}\left(\left(\chi^{\prime} \mid 1\right)^{-1}\right) \geq \sigma_{k}^{2}\left(\chi^{-1} \mid 1\right)
$$

Now suppose $\chi_{1} \geq N \chi_{n}$, and $\chi_{n} \geq \delta$. Then

$$
\begin{aligned}
\frac{\sigma_{k}\left(\chi^{-1} \mid 1\right)}{\sigma_{k}\left(\chi^{-1}\right)} & =1-\frac{\frac{1}{\chi_{1}} \sigma_{k-1}\left(\chi^{-1} \mid 1\right)}{\sigma_{k}\left(\chi^{-1}\right)} \\
& \geq 1-\frac{\frac{1}{\chi_{1}} \frac{\left(\begin{array}{c}
n-1 \\
k-1
\end{array}\right)}{\delta^{k-1}}}{\sigma_{k}\left(\chi^{-1}\right)} \geq 1-\frac{\left(\begin{array}{c}
n-1 \\
k-1
\end{array}\right)}{C_{1} N \delta^{k}} .
\end{aligned}
$$


Combine $(2.10),(2.12),(2.14)$, for $\theta$ sufficiently small, a positive number $N=\frac{\left(\begin{array}{l}n-1 \\ k-1\end{array}\right)}{C_{1} \delta^{k}} \frac{1}{1-(1+\theta)^{\frac{1}{2}}\left(\frac{c-\eta}{c}\right)}$ will satisfy the condition of this Proposition.

\section{Partial Second order estimate}

In this section, we use the maximum principle to obtain an estimate on the second order derivatives of $\varphi$ in terms of $\varphi$.

First we establish the ellipticity condition. Notice that by the basic properties of symmetric polynomials, $\left(F^{i \bar{j}}\right)>0$ if $\chi>0$. Differentiating (1.4) with respect to $t$ gives

$$
\frac{\partial}{\partial t}\left(\frac{\partial \varphi}{\partial t}\right)=F^{i \bar{j}}(\chi) \partial_{i} \partial_{\bar{j}}\left(\frac{\partial \varphi}{\partial t}\right)
$$

Standard theory for parabolic equation ensures short time existence of the flow. By the maximum principle, $\frac{\partial \varphi}{\partial t}$ achieves extremal values at $t=0$, i.e.

$$
\min _{t=0} \frac{\partial \varphi}{\partial t} \leq \frac{\partial \varphi}{\partial t} \leq \max _{t=0} \frac{\partial \varphi}{\partial t}
$$

which in terms implies

$$
\inf _{M} \frac{\sigma_{n-k}}{\sigma_{n}}\left(\chi_{0}\right) \leq \frac{\sigma_{n-k}}{\sigma_{n}}\left(\chi_{\varphi}\right) \leq \sup _{M} \frac{\sigma_{n-k}}{\sigma_{n}}\left(\chi_{0}\right) .
$$

Hence, $\chi_{\varphi}>0$, i.e., it remains Kähler when the flow exists.

Next we prove the partial $C^{2}$ estimate:

Theorem 3.1. Let $M, \omega$, and $\chi_{0} \in[\chi]$ as above. $k$ is an integer in $[1, n]$. Suppose $[\chi] \in \mathcal{C}_{k}$, i.e. there exists $\chi^{\prime} \in[\chi]$ such that:

$$
\chi^{\prime n-1}-\frac{n-k}{n} \omega^{k} \wedge \chi^{\prime n-k-1}>0 .
$$

Let $\varphi$ be a solution of (1.4) on $[0, T)$. Then there exist constants $A>0, C>0$, depending only on the initial data and independent of $T$, such that for any time $t \geq 0$,

$$
\|\partial \bar{\partial} \varphi\|_{C^{0}} \leq C e^{A\left(\varphi-\inf _{M \times[0, t]} \varphi\right)} .
$$

Proof. By hypothesis, there exists $\phi \in \mathcal{P}_{\chi_{0}}$, such that $\chi^{\prime}=\chi_{0}+\frac{\sqrt{-1}}{2} \partial \bar{\partial} \phi$, then $\chi_{\varphi}=$ $\chi^{\prime}+\frac{\sqrt{-1}}{2} \partial \bar{\partial}(\varphi-\phi)$. Consider the function

$$
G(x, t, \xi):=\log \left(\chi_{i \bar{j}} \xi^{i} \xi^{\bar{j}}\right)-A(\varphi-\phi),
$$

for $x \in M$, and $\xi \in \mathbf{T}_{x}^{(1,0)} M, g_{i \bar{j}} \xi^{i} \xi^{\bar{j}}=1$. $A$ is a constant to be determined. Fix a time $t$, we can assume $G$ attains maximum at $\left(x_{0}, t_{0}\right) \in M \times[0, t]$, along the direction $\xi_{0}$. Choose normal coordinates of $\omega$ at $x_{0}$, so that $\xi_{0}=\frac{\partial}{\partial z_{1}}$ and $\left(\chi_{i \bar{j}}\right)$ is diagonal at $x_{0}$. By the definition of $G$, it is easy to see that $\chi_{1 \overline{1}}=\chi_{1}$ is the largest eigenvalue of $\left\{\chi_{i \bar{j}}\right\}$ at $x_{0}$. Without loss of generality, we can assume $t_{0}>0$. Thus, locally, we consider $H:=\log \chi_{1 \overline{1}}-A(\varphi-\phi)$ 
instead, which also attains maximum at $\left(x_{0}, t_{0}\right)$, with $H\left(x_{0}, t_{0}\right)=G\left(x_{0}, t_{0}\right)$. We compute the evolution of $H$, namely the quantity $\frac{\partial H}{\partial t}-F^{i \bar{j}} H_{i \bar{j}}$. Then at $\left(x_{0}, t_{0}\right)$, we have

$$
\begin{gathered}
\frac{\partial H}{\partial t}=\frac{\chi_{1 \overline{1}, t}}{\chi_{1 \overline{1}}}-A \frac{\partial \varphi}{\partial t}, \\
H_{i \bar{i}}=\frac{\chi_{1 \overline{1}, \bar{i} \bar{i}}}{\chi_{1 \overline{1}}}-\frac{\left|\chi_{1 \overline{1}, i}\right|^{2}}{\chi_{1 \overline{1}}^{2}}-A\left(\varphi_{i \bar{i}}-\phi_{i \bar{i}}\right) .
\end{gathered}
$$

Take two derivatives along $\frac{\partial}{\partial z_{1}}$ direction to the equation (1.4), one gets

$$
\chi_{1 \overline{1}, t}=\left(\frac{\partial \varphi}{\partial t}\right)_{1 \overline{1}}=\sum_{i=1}^{n} F^{i \bar{i}} \chi_{i \bar{i}, 1 \overline{1}}+\sum_{1 \leq i, j, k, l \leq n} F^{i \bar{j}, k \bar{l}} \chi_{i \bar{j}, 1} \chi_{k \bar{l}, \overline{1}} .
$$

Apply (1.4), (3.4), (3.5), (3.6) we have, at $\left(x_{0}, t_{0}\right)$

$$
\begin{aligned}
& \frac{\partial H}{\partial t}-\sum_{i=1}^{n} F^{i \bar{i}} H_{i \bar{i}} \\
= & \frac{1}{\chi_{1 \overline{1}}}\left(\sum_{i=1}^{n} F^{i \bar{i}} \chi_{i \bar{i}, 1 \overline{1}}+\sum_{1 \leq i, j, k, l \leq n} F^{i \bar{j}, k \bar{l}} \chi_{i \bar{j}, 1} \chi_{k \bar{l}, \overline{1}}\right)-A \frac{\partial \varphi}{\partial t}-\sum_{i=1}^{n} F^{i \bar{i}} H_{i \bar{i}} \\
= & \frac{1}{\chi_{1 \overline{1}}} \sum_{i=1}^{n} F^{i \bar{i}}\left(\chi_{i \bar{i}, 1 \overline{1}}-\chi_{1 \overline{1}, i \bar{i}}\right)-A \frac{\partial \varphi}{\partial t}+A \sum_{i=1}^{n} F^{i \bar{i}}\left(\varphi_{i \bar{i}}-\phi_{i \bar{i}}\right)+B \\
= & \frac{1}{\chi_{1 \overline{1}}} \sum_{i=1}^{n} F^{i \bar{i}}\left(\chi_{i \bar{i}, 1 \overline{1}}-\chi_{1 \overline{1}, i \bar{i}}\right)-A\left(c^{\frac{1}{k}}+F\right)+A \sum_{i=1}^{n} F^{i \bar{i}}\left(\chi_{i \bar{i}}^{\prime}+\varphi_{i \bar{i}}-\phi_{i \bar{i}}\right)-A \sum_{i=1}^{n} F^{i \bar{i}} \chi_{i \bar{i}}^{\prime}+B \\
= & \frac{1}{\chi_{1}} \sum_{i=1}^{n} F^{i \bar{i}}\left(\chi_{i \bar{i}, 1 \overline{1}}-\chi_{1 \overline{1}, i \bar{i}}\right)-A c^{\frac{1}{k}}-2 A F-A \sum_{i=1}^{n} F^{i \bar{i}} \chi_{i \bar{i}}^{\prime}+B,
\end{aligned}
$$

where

$$
B=\frac{1}{\chi_{1 \overline{1}}} \sum_{1 \leq i, j, k, l \leq n} F^{i \bar{j}, k \bar{l}} \chi_{i \bar{j}, 1} \chi_{k \bar{l}, \overline{1}}+\sum_{i=1}^{n} F^{i \bar{i}} \frac{\left|\chi_{1 \overline{1}, i}\right|^{2}}{\chi_{1 \overline{1}}^{2}}
$$

includes all the third order derivatives terms of $\varphi$.

We claim that $B \leq 0$, the proof of which we postpone to the end of this section. By maximum principle, $\frac{\partial H}{\partial t}-\sum_{i=1}^{n} F^{i \bar{i}} H_{i \bar{i}} \geq 0$ at $\left(x_{0}, t_{0}\right)$, thus

$$
\frac{1}{\chi_{1 \overline{1}}} \sum_{i=1}^{n} F^{i \bar{i}}\left(\chi_{i \bar{i}, 1 \overline{1}}-\chi_{1 \overline{1}, i \bar{i}}\right)-A c^{\frac{1}{k}}-2 A F-A \sum_{i=1}^{n} F^{i \bar{i}} \chi_{i \bar{i}}^{\prime} \geq 0
$$

i.e.

$$
\begin{aligned}
\frac{1}{\chi_{1 \overline{1}}} \sum_{i=1}^{n} F^{i \bar{i}}\left(\chi_{i \bar{i}, 1 \overline{1}}-\chi_{1 \overline{1}, i \bar{i}}\right) & \geq A \sum_{i=1}^{n} F^{i \bar{i}} \chi_{i \bar{i}}^{\prime}+A c^{\frac{1}{k}}+2 A F \\
& \geq A \sum_{i=1}^{n} F^{i \bar{i}} \chi_{i \bar{i}}^{\prime}-A c^{-\frac{1}{k}} F^{2} \\
& =A \sum_{i=1}^{n} F^{i \bar{i}} \chi_{i \bar{i}}^{\prime}-A c^{-\frac{1}{k}} \sigma_{k}^{\frac{2}{k}}\left(\chi^{-1}\right) .
\end{aligned}
$$


Notice that

$$
\chi_{1 \overline{1}, i \bar{i}}=\chi_{i \bar{i}, 1 \overline{1}}+\chi_{1 \overline{1}} R_{1 \overline{1}, i \bar{i}}-\chi_{i \bar{i}} R_{i \bar{i}, 1 \overline{1}},
$$

so the left hand side of (3.7) can be simplified as follows

$$
\begin{aligned}
\frac{1}{\chi_{1 \overline{1}}} \sum_{i=1}^{n} F^{i \bar{i}}\left(\chi_{i \bar{i}, 1 \overline{1}}-\chi_{1 \overline{1}, i \bar{i}}\right) & =\frac{1}{\chi_{1 \overline{1}}} \sum_{i=1}^{n} F^{i \bar{i}}\left(\chi_{i \bar{i}} R_{i \bar{i} \overline{1} \overline{1}}-\chi_{1 \overline{1}} R_{1 \overline{1} i \bar{i}}\right) \\
& =\frac{1}{\chi_{1 \overline{1}}} \sum_{i=1}^{n} F^{i \bar{i}} \chi_{i \bar{i}} R_{i \bar{i} 1 \overline{1}}-\frac{1}{\chi_{1 \overline{1}}} \sum_{i=1}^{n} F^{i \bar{i}} \chi_{1 \overline{1}} R_{1 \overline{1}} \bar{i} \bar{i} \\
& \leq \frac{-C_{1} F}{\chi_{1 \overline{1}}}-\sum_{i=1}^{n} F^{i \bar{i}} R_{1 \overline{1} i \bar{i}} \\
& \leq \frac{C_{0}}{\chi_{1 \overline{1}}}+C_{2} \sum_{i=1}^{n} F^{i \bar{i}}
\end{aligned}
$$

where $C_{1}=\max \left\{1, \sup _{i, j}\left\{R_{i \bar{i} j \bar{j}}\right\}\right\},-C_{2}=\min \left\{-1, \inf _{i, j}\left\{R_{i \bar{i} j \bar{j}}\right\}\right\}$ are upper and lower bound of holomorphic bisectional curvature of $M$, and $C_{0}=C_{1} \sup _{M}\left[-F\left(\chi_{0}\right)\right]$. All constants here are positive.

Let $\chi_{1} \geq \cdots \geq \chi_{n}$ be the eigenvalues of $\chi$ with respect to $\omega$. Our goal is to get a uniform upper bound for $\chi_{1}=\chi_{1 \overline{1}}$.

If $k<n$, we have two cases:

Case 1. $\frac{\chi_{1}}{\chi_{n}} \leq N . N$ is the constant in Theorem 2.8. From (3.3), it follows that there exists a constant $C_{3}$ such that

$$
C_{3} \leq \sigma_{k}\left(\chi^{-1}\right) \leq \frac{\left(\begin{array}{l}
n \\
k
\end{array}\right)}{\chi_{n}^{k}},
$$

from which we get an upper bound

$$
\chi_{n} \leq\left(\frac{\left(\begin{array}{l}
n \\
k
\end{array}\right)}{C_{3}}\right)^{\frac{1}{k}}
$$

Hence

$$
\chi_{1} \leq N \chi_{n} \leq C
$$

for some uniform constant $C$.

Case 2. $\frac{\chi_{1}}{\chi_{n}} \geq N$. Then by Theorem 2.8, there exists $\epsilon>0$ such that

$$
\sum_{i=1}^{n} F^{i \bar{i}} \chi_{i \bar{i}}^{\prime}-c^{-\frac{1}{k}} \sigma_{k}^{\frac{2}{k}}\left(\chi^{-1}\right) \geq \epsilon \sum_{i=1}^{n} F^{i \bar{i}} \chi_{i \bar{i}}^{\prime} .
$$

Since $\chi^{\prime}$ is fixed and $M$ is compact, there exists $\gamma>0$, such that

$$
\epsilon \sum_{i=1}^{n} F^{i \bar{i}} \chi_{i \bar{i}}^{\prime} \geq \gamma \sum_{i=1}^{n} F^{i \bar{i}}
$$


Combine (3.7), (3.8), (3.9) and (3.10), we get

$$
\frac{C_{0}}{\chi_{1}}+C_{2} \sum_{i=1}^{n} F^{i \bar{i}} \geq A \gamma \sum_{i=1}^{n} F^{i \bar{i}}
$$

Since $\gamma>0$, we can choose $A$ so that $A \gamma-C_{2}=1$. Hence,

$$
\frac{C_{0}}{\chi_{1}} \geq \sum_{i=1}^{n} F^{i \bar{i}}
$$

Apply Gårding's Inequality, Cauchy inequality and (3.3), we have

$$
\begin{aligned}
\sum_{j=1}^{n} F^{j \bar{j}} & =\sum_{j=1}^{n} \frac{1}{k} \sigma_{k}^{\frac{1}{k}-1}\left(\chi^{-1}\right) \sigma_{k-1}\left(\chi^{-1} \mid j\right) \frac{1}{\chi_{j}^{2}} \\
& \geq \sigma_{k}^{\frac{1}{k}-1}\left(\chi^{-1}\right) \sigma_{k}^{1-\frac{1}{k}}\left(\chi^{-1}\right) \sigma_{k}^{\frac{1}{k}}\left(\chi^{-2}\right) \\
& \geq \frac{\sigma_{k}^{\frac{2}{k}}\left(\chi^{-1}\right)}{\left(\begin{array}{l}
n \\
k
\end{array}\right)} \geq \frac{C_{3}^{\frac{2}{k}}}{\left(\begin{array}{l}
n \\
k
\end{array}\right)} .
\end{aligned}
$$

Combine (3.12) and (3.13), we have

$$
\chi_{1} \leq C,
$$

for some constant $C$ depending only on the initial data.

For $k=n$, notice in this case $c=1$. From Proposition 2.1,

$$
\sum_{i=1}^{n} F^{i \bar{i}}=\frac{1}{n} \sigma_{n}^{-\frac{1}{n}}(\chi) \sum_{i=1}^{n} \frac{1}{\chi_{i}} .
$$

By (3.3), there exists two positive constants $C_{4}$ and $C_{5}$, such that

$$
0<C_{4} \leq \sigma_{n}^{-\frac{1}{n}}(\chi) \leq C_{5}<+\infty .
$$

Now we can proceed directly from (3.7) and (3.8), namely:

$$
A+2 A F+A \sum_{i=1}^{n} F^{i \bar{i}} \chi_{i \bar{i}}^{\prime} \leq \frac{C_{0}}{\chi_{1}}+C_{1} \sum_{i=1}^{n} F^{i \bar{i}} .
$$

Assume $\chi_{i \bar{i}}^{\prime} \geq \epsilon_{o}>0$. Using (3.15) it follows that

$$
A-2 A \sigma_{n}^{-\frac{1}{n}}(\chi)+\frac{A \epsilon_{o}}{n} \sigma_{n}^{-\frac{1}{n}}(\chi) \sum_{i=1}^{n} \frac{1}{\chi_{i}} \leq \frac{C_{0}}{\chi_{1}}+C_{6} \sum_{i=1}^{n} \frac{1}{\chi_{i}} \leq C_{7} \sum_{i=1}^{n} \frac{1}{\chi_{i}} .
$$

Apply (3.15) again, we get

$$
\left(\frac{A \epsilon_{o}}{n} C_{4}-C_{7}\right) \sum_{i=1}^{n} \frac{1}{\chi_{i}} \leq 2 A C_{5} .
$$

Now we take $A$ such that $\frac{A \epsilon_{o}}{n} C_{4}-C_{7}=1, \quad$ i.e., $\quad A=\frac{n\left(1+C_{7}\right)}{\epsilon_{o} C_{4}}$. From (3.18), we have

$$
\sum_{i=1}^{n} \frac{1}{\chi_{i}} \leq C_{8} .
$$


Since $\chi_{i}>0$

$$
\chi_{i} \geq C_{8}^{-1}
$$

Combining (3.15) and (3.19), it follows that there exists a uniform constant $C_{9}$

$$
\chi_{1}=\frac{\left(\Pi_{i=2}^{n} \chi_{i}^{-1}\right)}{\sigma_{n}\left(\chi^{-1}\right)} \leq C_{8}^{n-1} / C_{4}^{n}=C,
$$

for a uniform constant $C$.

In summary, for all $1 \leq k \leq n$, there exists a uniform constant $C$, such that $\chi_{1} \leq C$. Back in the definition of $\mathrm{G}$, we have

$$
\log \left(\chi_{i \bar{j}}\right)-A(\varphi-\phi) \leq \log \left(\chi_{1}\left(x_{0}\right)\right)-A\left(\varphi\left(x_{0}\right)-\phi\left(x_{0}\right)\right),
$$

so

$$
\log \left(\chi_{i \bar{j}}\right) \leq \log C-A \varphi\left(x_{0}\right)+A \varphi+C^{\prime} .
$$

Exponentiating both sides, we get the desired estimate.

Now we prove the claim: $B=\frac{1}{\chi_{1 \overline{1}}} \sum_{i, j, k, l} F^{i \bar{j}, k \bar{l}} \chi_{i \bar{j}, 1} \chi_{k \bar{l}, \overline{1}}+\sum_{i} F^{i \bar{i}} \frac{\left|\chi_{1 \overline{1}, i}\right|^{2}}{\chi_{1 \overline{1}}^{2}} \leq 0$.

Proof. Case 1. $k<n$.

Recall from Proposition 2.1, $F^{i \bar{j}, k \bar{l}}$ is not zero iff $i=j, k=l$ or $i=l, k=j$. According to the computation there, we have for $i \neq j$

$$
\begin{aligned}
F^{i \bar{j}, j \bar{i}} & =\frac{1}{k}\left(\frac{\sigma_{n-k}(\chi)}{\sigma_{n}(\chi)}\right)^{\frac{1}{k}-1}\left(\frac{\sigma_{n} \sigma_{n-k-2}(\chi \mid i, j)-\sigma_{n-k} \sigma_{n-2}(\chi \mid i, j)}{\sigma_{n}^{2}}\right) \\
& =-\frac{1}{k}\left(\frac{\sigma_{n-k}(\chi)}{\sigma_{n}(\chi)}\right)^{\frac{1}{k}-1}\left(\frac{\chi_{i} \sigma_{n-k-1}(\chi \mid i, j)+\chi_{j} \sigma_{n-k-1}\left(\chi_{j} \mid i, j\right)+\chi_{i} \chi_{j} \sigma_{n-k-2}(\chi \mid i, j)}{\sigma_{n}^{2}}\right) \\
(3.22) & <0 .
\end{aligned}
$$

So we group terms as follows:

The first group:

$$
X=\frac{1}{\chi_{1 \overline{1}}}\left(\sum_{1 \leq i, j \leq n} F^{i \bar{i}, j \bar{j}} \chi_{i \bar{i}, 1} \chi_{j \bar{j}, \overline{1}}\right)+F^{1 \overline{1}} \frac{\left|\chi_{1 \overline{1}, 1}\right|^{2}}{\chi_{1 \overline{1}}^{2}} \leq 0 .
$$

Let

$$
f(\chi)=-\left(\frac{\sigma_{n-k}}{\sigma_{n}}\right)^{\frac{1}{k}}(\chi) .
$$

It is sufficient to prove the following point-wise matrix inequality:

$$
\left(f_{\chi_{i} \chi_{j}}+\frac{f_{\chi_{i}}}{\chi_{j}} \delta_{i j}\right) \leq 0 .
$$

If we let $\lambda_{i}=\frac{1}{\chi_{i}}$, and $g(\lambda)=\sigma_{k}^{\frac{1}{k}}(\lambda)$, then (3.23) is equivalent to the following

$$
\left(g_{\lambda_{i} \lambda_{j}}+\frac{g_{\lambda_{i}}}{\lambda_{i}} \delta_{i j}\right) \geq 0
$$

which is true by Proposition 2.2 and Remark 2.3. See also Appendix for an alternative proof. 
Second group:

$$
Y=\frac{1}{\chi_{1} \overline{1}} \sum_{i=2}^{n} F^{i \overline{1}, 1 \bar{i}} \chi_{i \overline{1}, 1} \chi_{1 \bar{i}, \overline{1}}+\sum_{i=2}^{n} F^{i \bar{i}} \frac{\left|\chi_{1 \overline{1}, i}\right|^{2}}{\chi_{1 \overline{1}}^{2}} \leq 0 .
$$

The idea is to use $F^{i \overline{1}, 1 \bar{i}}$ to control $F^{i \bar{i}}$, take $i=2$ for example. By the Kähler property of $\chi$, we have:

$$
\chi_{i \bar{j}, k}=\chi_{k \bar{j}, i}, \chi_{i \bar{j}, \bar{k}}=\chi_{i \bar{k}, \bar{j}}
$$

It suffices to show

$$
\chi_{1 \overline{1}} F^{j \overline{1}, 1 \bar{j}}+F^{j \bar{j}} \leq 0, j \neq 1 .
$$

After taking out the common factor $\frac{1}{k \sigma_{n}^{2}(\chi)}\left(\frac{\sigma_{n-k}(\chi)}{\sigma_{n}(\chi)}\right)^{\frac{1}{k}}$, we are left to show $\chi_{1}\left[\sigma_{n}(\chi) \sigma_{n-k-2}(\chi \mid 1, j)-\sigma_{n-k}(\chi) \sigma_{n-2}(\chi \mid 1, j)\right]+\sigma_{n-k}(\chi) \sigma_{n-1}(\chi \mid j)-\sigma_{n-k-1}(\chi \mid j) \sigma_{n}(\chi) \leq 0$.

Here we simply write $\chi_{1}$ for $\chi_{1 \overline{1}}$. Use the identity $\sigma_{k}(\chi)=\sigma_{k}(\chi \mid 1)+\chi_{1} \sigma_{k-1}(\chi \mid 1)$, we have

$$
\begin{aligned}
& \chi_{1}\left[\sigma_{n}(\chi) \sigma_{n-k-2}(\chi \mid 1, j)-\sigma_{n-k}(\chi) \sigma_{n-2}(\chi \mid 1, j)\right]+\sigma_{n-k}(\chi) \sigma_{n-1}(\chi \mid j)-\sigma_{n-k-1}(\chi \mid j) \sigma_{n}(\chi) \\
= & \sigma_{n}(\chi)\left[\chi_{1} \sigma_{n-k-1}(\chi \mid j)-\sigma_{n-k-1}(\chi \mid j)\right]-\sigma_{n-k}(\chi)\left[\chi_{1} \sigma_{n-2}(\chi \mid 1, j)-\sigma_{n-1}(\chi \mid j)\right] \\
= & -\sigma_{n}(\chi) \sigma_{n-k-1}(\chi \mid 1,2) \leq 0 .
\end{aligned}
$$

The third group have all the remaining terms:

$$
Z=\frac{1}{\chi_{1 \overline{1}}} \sum_{1 \leq i \leq n, 2 \leq j \leq n, i \neq j} F^{i \bar{j}, j \bar{i}} \chi_{i \bar{j}, 1} \chi_{j \bar{i}, \overline{1}} \leq 0 .
$$

By (3.22), each term in $Z$ is negative.

To sum up, we have

$$
B=X+Y+Z \leq 0
$$

Case 2. $k=n$.

If we use the convention $\sigma_{-1}(\chi)=0$, the computation above is valid and can be simplied.

\section{Convergence of the flow}

In this section, we study the properties of the functionals $\tilde{\mathcal{F}}_{k, n}$ raised in the introduction, from which we prove the uniqueness of the solution of (1.5) and $C^{0}$ estimate for the oscillation of $\varphi_{t}$. After getting $C^{0}$ estimate of oscillation of $\varphi_{t}$, all the arguments in [W2] can be applied verbatim.

For any $\phi \in \mathcal{P}_{\chi_{0}}$, let

$$
\delta \mathcal{F}_{k}(\phi)=\int_{M} \delta \phi \chi_{\phi}^{k} \wedge \omega^{n-k}
$$

be the infinitesimal variation of the functional $\mathcal{F}_{k}$. Then one has explicit formula for $\mathcal{F}_{k}$ :

$$
\mathcal{F}_{k}(\phi)=\int_{0}^{1} \int_{M} \dot{\phi}_{t} \chi_{\phi_{t}}^{k} \wedge \omega^{n-k} d t,
$$


where $\phi_{t}$ is an arbitrary path in $\mathcal{P}_{\chi_{0}}$ connecting 0 and $\phi$, and $\dot{\phi}_{t}$ denotes time derivative. Then let:

$$
\tilde{\mathcal{F}}_{k, n}(\phi)=\mathcal{F}_{k}(\phi)-c_{n-k} \mathcal{F}_{n}(\phi)
$$

By the variational characterization of (4.1), one has

$$
\delta \tilde{\mathcal{F}}_{n-k, n}(\phi)=\int_{M} \delta \phi\left(\chi_{\phi}^{n-k} \wedge \omega^{k}-c_{k} \chi \phi^{n}\right) .
$$

So the Euler-Lagrange equation of $\tilde{\mathcal{F}}_{n-k, n}$ is

$$
\chi_{\phi}^{n-k} \wedge \omega^{k}-c_{k} \chi_{\phi}^{n}=0
$$

which is exactly (1.5). Regarding the second derivative of $\tilde{\mathcal{F}}_{k, n}$, one chooses a path $\phi_{t}$ and use (4.1), (4.2) to get:

$$
\begin{aligned}
\frac{d^{2} \tilde{\mathcal{F}}_{n-k, n}\left(\phi_{t}\right)}{d t^{2}} & =\int_{M} \ddot{\phi}_{t}\left(\chi_{\phi}^{n-k} \wedge \omega^{k}-c_{k} \chi_{\phi}^{n}\right)+\int_{M} \dot{\phi}_{t} \partial \bar{\partial} \dot{\phi}_{t}\left((n-k) \chi_{\phi}^{n-k-1} \wedge \omega^{k}-c_{k} n \chi_{\phi}^{n-1}\right) \\
(4.5) & =\int_{M} \ddot{\phi}_{t}\left(\chi_{\phi}^{n-k} \wedge \omega^{k}-c_{k} \chi_{\phi}^{n}\right)+\int_{M} \partial \dot{\phi}_{t} \wedge \bar{\partial} \dot{\phi}_{t}\left(c_{k} n \chi_{\phi}^{n-1}-(n-k) \chi_{\phi}^{n-k-1} \wedge \omega^{k}\right)
\end{aligned}
$$

We observe the following

Theorem 4.1. There is only one critical point at the level of Kähler metric if such critical point exists.

Proof. Suppose we have two critical points $\phi_{0}$ and $\phi_{1}$. Consider the affine path $\phi_{t}=$ $(1-t) \phi_{0}+t \phi_{1}, t \in[0,1] . \phi_{0}$ and $\phi_{1}$ being critical points are equivalent, in local coordinates, to following inequalities

$$
\sigma_{k}\left(\chi_{\phi_{0}}^{-1}\right)=\sigma_{k}\left(\chi_{\phi_{1}}^{-1}\right)=c_{k}^{\prime} .
$$

Recall that in Section 2, we have proved $-\sigma_{k}\left(\chi^{-1}\right)=F$ is concave, which is equivalent to the convexity of $\sigma_{k}\left(\chi^{-1}\right)$. Thus

$$
\sigma_{k}\left(\chi_{\phi_{t}}^{-1}\right) \leq(1-t) c_{k}^{\prime}+t c_{k}^{\prime}=c_{k}^{\prime}, \quad t \in[0,1]
$$

Since $\chi_{\phi_{t}}^{-1}$ is positive definite, we have $\sigma_{k}\left(\chi_{\phi_{t}}^{-1} \mid i\right)<c_{k}^{\prime}$. By Proposition 2.4, it follows

$$
c_{k} n \chi_{\phi_{t}}^{n-1}-(n-k) \chi_{\phi_{t}}^{n-k-1}>0
$$

as a $(n-1, n-1)$ form. Therefore by (4.5) and the facts that $\dot{\phi}_{t}=\phi_{1}-\phi_{0}, \ddot{\phi}_{t}=0$, we conclude that $\tilde{\mathcal{F}}_{n-k, n}\left(\phi_{t}\right)$ is a convex function: $[0,1] \rightarrow \mathbb{R}$, with critical points at $t=0,1$. This implies that $\tilde{\mathcal{F}}_{n-k, n}\left(\phi_{t}\right)$ is a constant. Furthermore, the indentity

$$
\frac{d^{2} \tilde{\mathcal{F}}_{n-k, n}\left(\phi_{t}\right)}{d t^{2}}=0
$$

implies $\dot{\phi}_{t}=\phi_{1}-\phi_{0}=C$ for some constant $C$, hence $\chi_{\phi_{0}}=\chi_{\phi_{1}}$.

Next, we establish some propositions regarding monotonicity of the functionals which will lead to the $C^{0}$ estimates. 
Proposition 4.2. The functional $\tilde{\mathcal{F}}_{n-k, n}$ is decreasing along the flow (1.4).

Proof. We write (1.4) as

$$
\dot{\varphi}_{t}=\left(c_{k}^{\prime}\right)^{\frac{1}{k}}+F
$$

where $F=-\left(\frac{\sigma_{n-k}\left(\varphi_{t}\right)}{\sigma_{n}\left(\varphi_{t}\right)}\right)^{\frac{1}{k}}$.

$$
\begin{aligned}
\frac{d}{d t} \tilde{\mathcal{F}}_{n-k, n}\left(\varphi_{t}\right) & =\int_{M} \dot{\varphi}_{t}\left(\chi_{\varphi_{t}}^{n-k} \wedge \omega^{k}-c_{k} \chi_{\varphi}^{n}\right) \\
& =\frac{1}{\left(\begin{array}{l}
n \\
k
\end{array}\right)} \int_{M}\left(\left(c_{k}^{\prime}\right)^{1 / k}+F\right)\left(F^{k}-c_{k}^{\prime}\right) \chi_{\varphi_{t}}^{n} \leq 0
\end{aligned}
$$

The integrand is of the form $\left(a^{1 / k}-b^{1 / k}\right)(b-a)$ which is clearly non-positive.

Corollary 4.3. Assume the convergence of the flow, i.e., the existence of the solution of (1.5), then the global minimum of $\tilde{\mathcal{F}}_{n-k, n}$ is realized by the critical metric.

Proof. It follows directly from Proposition 4.1 and Proposition 4.2 .

Towards $C^{0}$ estimate, we need another monotonicity:

Proposition 4.4. Let $\mathcal{F}_{n-k}$ defined as above, $\varphi_{t}$ the solution of flow (1.4), then

$$
\frac{d \mathcal{F}_{n-k}\left(\varphi_{t}\right)}{d t} \leq 0
$$

i.e. $\mathcal{F}_{n-k}\left(\varphi_{t}\right)$ decreases along the flow. In particular, $\mathcal{F}_{n-k}\left(\varphi_{t}\right) \leq 0$ for all $t>0$.

Proof. First we make following observation:

$$
\begin{aligned}
\int_{M} \sigma_{n-k} d v & =\int_{M}\left(\frac{\sigma_{n-k}}{\left(\sigma_{n}\right)^{\frac{1}{k+1}}}\right)\left(\sigma_{n}\right)^{\frac{1}{k+1}} d v \\
& \leq\left[\int_{M}\left(\frac{\sigma_{n-k}}{\left(\sigma_{n}\right)^{\frac{1}{k+1}}}\right)^{\frac{1+k}{k}} d v\right]^{\frac{k}{k+1}}\left(\int_{M} \sigma_{n} d v\right)^{\frac{1}{1+k}} \\
& =\left(\int_{M} \frac{\left(\sigma_{n-k}\right)^{\frac{1+k}{k}}}{\left(\sigma_{n}\right)^{\frac{1}{k}}} d v\right)^{\frac{k}{k+1}}\left(\int_{M} \sigma_{n} d v\right)^{\frac{1}{1+k}}
\end{aligned}
$$

Recall $d v=\frac{\omega^{n}}{n !}$, so $\sigma_{n-k} d v=\frac{\left(\begin{array}{l}n \\ k\end{array}\right)}{n !} \chi^{n-k} \wedge \omega^{k}$. So (4.6) gives:

$$
\int_{M}\left(\frac{\sigma_{n-k}}{\sigma_{n}}\right)^{\frac{1}{k}} \chi^{n-k} \wedge \omega^{k} \geq c_{k}^{\prime \frac{1}{k}} \int_{M} \chi^{n-k} \wedge \omega^{k} .
$$

Now we compute $\frac{d}{d t} \mathcal{F}_{n-k}\left(\varphi_{t}\right)$ by choosing the path given by the flow then

$$
\begin{aligned}
\frac{d}{d t} \mathcal{F}_{n-k}\left(\varphi_{t}\right) & =\int_{M} \dot{\varphi}_{t} \chi_{\varphi_{t}}^{n-k} \wedge \omega^{k} \\
& =\int_{M}\left[c_{k}^{\prime 1 / k}+F\right] \chi_{\varphi_{t}}^{n-k} \wedge \omega^{k} \\
& =\int_{M} c_{k}^{\prime 1 / k} \chi_{\varphi_{t}}^{n-k} \wedge \omega^{k}-\int_{M}\left(\frac{\sigma_{n-k}}{\sigma_{n}}\right)^{\frac{1}{k}} \chi_{\varphi_{t}}^{n-k} \wedge \omega^{k} \leq 0 .
\end{aligned}
$$


From Proposition 4.4, we know $\mathcal{F}_{n-k}\left(\varphi_{t}\right) \leq 0$. But the definition of $\mathcal{F}_{n-k}$ is independent of the choice of the path, we can choose the path $\gamma(s)=s \varphi_{t}$ to compute $\mathcal{F}_{n-k}\left(\varphi_{t}\right)$ as well.

$$
\begin{aligned}
\mathcal{F}_{n-k}\left(\varphi_{t}\right) & =\int_{0}^{1} \int_{M} \varphi_{t} \chi_{s \varphi_{t}}^{n-k} \wedge \omega^{k} d s \\
& =\int_{0}^{1} \int_{M} \varphi_{t}\left(s \chi_{\varphi_{t}}+(1-t) \chi_{0}\right)^{n-k} \wedge \omega^{k} d s \\
& =\sum_{l=0}^{n-k} \int_{0}^{1}\left(\begin{array}{c}
n-k \\
l
\end{array}\right) s^{l}(1-s)^{n-k-l} d s \int_{M} \varphi_{t} \chi_{\varphi_{t}}^{l} \wedge \chi_{0}^{n-k-l} \wedge \omega^{k} \leq 0 .
\end{aligned}
$$

So at time $t$, we may write in short $\mathcal{F}_{n-k}\left(\varphi_{t}\right)=\int_{M} \varphi_{t} d \mu_{t}$. Now we are in the position to prove following:

Theorem 4.5. Suppose that $\chi^{\prime n-1}-\frac{n-k}{n} \omega^{k} \wedge \chi^{\prime n-k-1}>0$. Let $\varphi_{t}$ be a solution of (1.4) on $[0, \infty)$. Then there exists a constant $\tilde{C}$, depending only on initial data such that

$$
\left\|\sup \varphi_{t}-\inf \varphi_{t}\right\|_{C^{0}} \leq \tilde{C} .
$$

Proof. It suffices to show a uniform lower bound of inf $\tilde{\varphi}_{t}$, where $\tilde{\varphi}_{t}=\varphi_{t}-\sup _{M} \varphi_{t}$. Following [W2], we prove by contradiction. If such a lower bound does not exist, then we can choose a sequence of times $t_{i} \rightarrow \infty$ such that

- $\inf _{M} \tilde{\varphi}_{t_{i}}=\inf _{t \in\left[0, t_{i}\right]} \inf _{M} \tilde{\varphi}_{t}$

- $\inf _{M} \tilde{\varphi_{t}} \rightarrow-\infty$

Set $B=A /(1-\delta)$ where $\mathrm{A}$ is the constant in Theorem 3.1, and let $\delta$ be a small positive constant to be determined later. Let $u=e^{-B \tilde{t_{i}}}$. We apply Lemma 3.3, Lemma 3.4 of W2], there is a constant $c^{\prime}$ independent of $\mathrm{u}$,such that

$$
\|u\|_{C^{0}} \leq C^{\prime}\|u\|_{\delta}
$$

Since $u=e^{-B \tilde{t_{i}}}$ and $\tilde{\varphi_{t_{i}}}$ satisfies $\sup _{M} \tilde{\varphi_{t_{i}}}=0$ and

$$
\chi_{0 k \bar{l}}+\left(\tilde{\varphi_{t_{i}}}\right)_{k \bar{l}}=\chi_{k \bar{l}}>0
$$

we can apply Proposition 2.1 of [T1] to get a bound on $\|u\|_{\delta}$ for $\delta$ small enough. This gives the uniform $C^{0}$ estimate of $\tilde{\varphi}_{t}$.

So far we have got the uniform $C^{0}$ estimate for oscillation of $\varphi_{t}$, in order to get convergence we have to normalize $\varphi_{t}$, namely let

$$
\hat{\varphi}_{t}=\varphi_{t}-\frac{\mathcal{F}_{n-k}\left(\varphi_{t}\right)}{\int_{M} d \mu_{t}} .
$$

Then $\hat{\varphi}_{t}$ takes value zero somewhere, by Theorem 4.5, $\left\|\hat{\varphi}_{t}\right\|_{C^{0}} \leq \tilde{C}$. With this choice of normalization, we see the partial $C^{2}$ estimate is actually uniform. By Theorem 3.1

$$
\left\|\partial \bar{\partial} \hat{\varphi}_{t}\right\|_{C^{0}}=\left\|\partial \bar{\partial} \varphi_{t}\right\|_{C^{0}} \leq A e^{c\left(\varphi_{t}-\inf _{M \times[0, t]} \varphi_{t}\right)} .
$$


For the exponent, we have

$$
\begin{aligned}
\varphi_{t}-\inf _{M \times[0, t]} \varphi_{t} & =\hat{\varphi}_{t}+\frac{\mathcal{F}_{n-k}\left(\varphi_{t}\right)}{\int_{M} d \mu_{t}}-\inf _{M \times[0, t]}\left(\hat{\varphi}_{t}+\frac{\mathcal{F}_{n-k}\left(\varphi_{t}\right)}{\int_{M} d \mu_{t}}\right) \\
& \leq \hat{\varphi}_{t}+\frac{\mathcal{F}_{n-k}\left(\varphi_{t}\right)}{\int_{M} d \mu_{t}}-\inf _{M \times[0, t]} \hat{\varphi}_{t}-\inf _{M \times[0, t]} \frac{\mathcal{F}_{n-k}\left(\varphi_{t}\right)}{\int_{M} d \mu_{t}} \\
& =\hat{\varphi}_{t}-\inf _{M \times[0, t]} \hat{\varphi}_{t}+\frac{\mathcal{F}_{n-k}\left(\varphi_{t}\right)}{\int_{M} d \mu_{t}}-\inf _{M \times[0, t]} \frac{\mathcal{F}_{n-k}\left(\varphi_{t}\right)}{\int_{M} d \mu_{t}} \\
& =\hat{\varphi}_{t}-\inf _{M \times[0, t]} \hat{\varphi}_{t} \leq 2 \tilde{C} .
\end{aligned}
$$

Last equality follows from Proposition 4.4 and the fact $\int_{M} d \mu_{t}$ is independent of $t$. Hence, we have a uniform constant $C$ such that

$$
\left\|\partial \bar{\partial} \varphi_{t}\right\|_{C^{0}}<C
$$

Since we get bound for complex hessian of $\varphi$, the underlying real parabolic equation (1.4) has uniform elliptic constants. By Wang1, Wang2, one can deduce $C^{2, \alpha}$ spatial and time estimate on $\varphi$. Then classical Schauder theory can be applied to prove estimates all the way to $C^{\infty}$. Consequently the flow exists on $[0, \infty)$. We will provide more explanations of PDE aspect in Appendix B.

To show the convergence without passing to a subsequence, one can follow the methods in $[\mathrm{C}], \mathrm{W} 2$.

\section{Generalization and Applications}

In this section, we apply Theorem 3.1 to the product manifold $M \times C$, where $C$ is an algebraic curve, to prove Theorem 1.2 .

Proof. First, let us recall the definition following constants:

$$
\begin{gathered}
c_{k}=c_{k,[\omega],[\chi]}=\frac{\int_{M} \chi_{0}^{k} \wedge \omega^{n-k}}{\int_{M} \chi_{0}^{n}}, \\
c_{k, \alpha}=c_{k}+\alpha c_{k-1}, \quad \alpha \geq 0,
\end{gathered}
$$

and cone condition $\mathcal{C}_{k, \alpha}=\mathcal{C}_{k, \alpha}(\omega)$ :

$$
\begin{aligned}
\mathcal{C}_{k, \alpha}(\omega)= & \left\{[\chi] \in \mathcal{H}^{+}, \exists \chi^{\prime} \in[\chi],\right. \text { such that } \\
& \left.c_{k, \alpha} n \chi^{\prime n-1}>(n-k) \chi^{\prime n-k-1} \wedge \omega^{k}+\alpha(n-k+1) \chi^{\prime n-k} \wedge \omega^{k-1}\right\} .
\end{aligned}
$$

Let $\omega_{0}, \chi_{0} \in[\chi]$ be two Kähler forms on M, $\omega_{1}$ be a Kähler form on $C$. Set

$$
\tilde{\chi}_{0}=\chi_{0}+a \omega_{1}, \text { and } \tilde{\omega}_{0}=\omega_{0}+\omega_{1} .
$$

Then on $M \times C$, consider following flow in $\mathcal{P}_{\chi_{0}}$,

$$
\frac{\partial \varphi}{\partial t}=c^{\frac{1}{k}}-\left(\frac{\sigma_{n+1-k}\left(\tilde{\chi_{\varphi}}\right)}{\sigma_{n+1}\left(\tilde{\chi_{\varphi}}\right)}\right)^{\frac{1}{k}},\left.\quad \varphi\right|_{t=0}=0
$$


where $\tilde{\chi_{\varphi}}=\tilde{\chi}_{0}+\frac{\sqrt{-1}}{2} \partial \bar{\partial} \varphi$, and

$$
c=\frac{\int_{M \times C} \sigma_{n+1-k}\left(\tilde{\chi_{\varphi}}\right)}{\int_{M \times C} \sigma_{n+1}\left(\tilde{\chi_{\varphi}}\right)}=\frac{a \int_{M} \sigma_{n-k}\left(\chi_{0}\right)+\int_{M} \sigma_{n-k+1}\left(\chi_{0}\right)}{a \int_{M} \sigma_{n}\left(\chi_{0}\right)}=\left(\begin{array}{l}
n \\
k
\end{array}\right) c_{k}+\frac{1}{a}\left(\begin{array}{c}
n \\
k-1
\end{array}\right) c_{k-1} .
$$

In local coordinates, one shall view the matrix $\left(\left(\tilde{\chi}_{\varphi}\right)_{i \bar{j}}\right)$ as $\left(\begin{array}{cc}\left(\chi_{\varphi}\right)_{i \bar{j}} & 0 \\ 0 & a \omega_{1}\end{array}\right)$. In view of Theorem [3.1, we want to bound the largest eigenvalue of $\left(\begin{array}{cc}\left(\chi_{\varphi}\right)_{i \bar{j}} & 0 \\ 0 & a \omega_{1}\end{array}\right)$. Without loss of generality, we can assume the corresponding direction is $\frac{\partial}{\partial z_{1}} \in T^{(1,0)} M$. Otherwise the estimate follows trivially, since $\omega_{1}$ is fixed under the flow. Compare the proof of Theorem 3.1. we impose condition:

$$
\sigma_{k}\left(\tilde{\chi}_{0}^{-1} \mid i\right)<c, \quad \forall i=1,2, \cdots, n .
$$

which translates to a condition on $M$ as:

$$
\frac{1}{a} \sigma_{k-1}\left(\chi_{0}^{-1} \mid i\right)+\sigma_{k}\left(\chi_{0}^{-1} \mid i\right)<c, \quad \forall i=1,2, \cdots, n .
$$

Then the whole argument applies. Moreover, $C^{0}$ estimate can be applied directly. Therefore we get a stationary metric $\chi$ on $M$ solving:

$$
a c \chi^{n}=a\left(\begin{array}{l}
n \\
k
\end{array}\right) \chi^{n-k} \wedge \omega^{k}+\left(\begin{array}{c}
n \\
k-1
\end{array}\right) \chi^{n-k+1} \wedge \omega^{k-1} .
$$

After setting $\alpha=\frac{\left(\begin{array}{c}n \\ k-1\end{array}\right)}{a\left(\begin{array}{c}n \\ k\end{array}\right)}$, one can readily check that $[\chi] \in \mathcal{C}_{k, \alpha}$ imply (5.5), and (5.7) becomes

$$
c_{k, \alpha} \chi^{n}=\chi^{n-k} \wedge \omega^{k}+\alpha \chi^{n-k+1} \wedge \omega^{k-1} .
$$

Based on the known result, we can refine Chen's problem into the following:

Conjecture 5.1. For fixed $q, 0 \leq q \leq n$, and for any given $\alpha=\left(\alpha_{0}, \cdots, \alpha_{p}\right) \in \mathbb{R}^{p+1}, p \leq$ $n-q \alpha_{i}>0,0 \leq i \leq p$, define

$$
\begin{aligned}
c_{\alpha} & =c_{k, \alpha,[\omega],[\chi]}=\sum_{i=0}^{p} c_{i+q} \alpha_{i}, \\
\tilde{\mathcal{F}}_{\alpha, n}\left(\chi_{0}, \chi\right) & =\sum_{i=0}^{p} \alpha_{i} \tilde{\mathcal{F}}_{i+q, n}\left(\chi_{0}, \chi\right), \\
\mathcal{C}_{\alpha}(\omega) & =\left\{[\chi] \in \mathcal{H}^{+}, \exists \chi^{\prime} \in[\chi], \text { such that } c_{\alpha} n \chi^{\prime n-1}>\sum_{i=0}^{p} \alpha_{i}(n-i-q) \chi^{\prime n-i-q-1} \wedge \omega^{i+q}\right\} .
\end{aligned}
$$

Then

$$
c_{\alpha} \chi_{\varphi}^{n}=\sum_{i=0}^{p} \alpha_{i} \chi_{\varphi}^{i+q} \wedge \omega^{n-i-q}
$$


has a unique smooth solution if and only if $[\chi] \in \mathcal{C}_{\alpha}(\omega)$; in this case, $\tilde{\mathcal{F}}_{\alpha, n}\left(\chi_{0}, \chi\right)$ obtains minimal at the given solution.

Use the same method we can verify Conjecture 5.1 under some additional conditions on $\alpha_{i}$ 's. We consider $M \times C_{1} \times C_{2} \cdots \times C_{p}$, where $C_{i}$ are all algebraic curves. Set $\omega_{i}$ be Kähler forms on $C_{i}$. For $a_{i}>0$ set

$$
\tilde{\chi}_{0}=\chi_{0}+\sum_{i=1}^{p} a_{i} \omega_{i}, \quad \tilde{\omega}=\sum_{i=0}^{n} \omega_{i} .
$$

Follow the method above one can solve

$$
c \sigma_{n+p}(\tilde{\chi})=\sigma_{n+p-k}(\tilde{\chi}), \text { on } \tilde{M}:=M \times C_{1} \times C_{2} \cdots \times C_{p},
$$

where $c$ is the constant satisfying

$$
c=\frac{\int_{\tilde{M}} \sigma_{n+p-k}(\tilde{\chi})}{\int_{\tilde{M}} \sigma_{n+p}(\tilde{\chi})}
$$

Similarly, one reduces (5.9) to an equation on $M$. According to the relationship of $k, n$, and $p$, there will be four cases which we state as a theorem.

Theorem 5.2. Let $M, \omega$, and $[\chi]$ be as above. $\Gamma_{p}$ is the positive cone in $\mathbb{R}^{p}$. Conjecture 5.1 holds for the following special equations:

(1) For $p \geq k$ and $n>k$,

$$
c \chi^{n}=\beta_{0} \chi^{n}+\beta_{1} \chi^{n-1} \wedge \omega+\cdots+\beta_{k} \chi^{n-k} \wedge \omega^{k}, c=\sum_{i=0}^{k} \beta_{i} c_{i},
$$

for which we require the existence of $a b=\left(b_{1}, b_{2}, \cdots, b_{p}\right) \in \Gamma_{p}$ such that $\beta_{i}=$ $\sigma_{k-i}(b)\left(\begin{array}{c}n \\ i\end{array}\right), i=0,1, \cdots k$

(2) For $p<k<n$,

$c \chi^{n}=\beta_{0} \chi^{n+p-k} \wedge \omega^{k-p}+\beta_{1} \chi^{n+p-k-1} \wedge \omega^{k-p+1}+\cdots+\beta_{p} \chi^{n-k} \wedge \omega^{k}, c=\sum_{i=0}^{p} \beta_{i} c_{k-p+i}$,

for which we require the existence of $a b=\left(b_{1}, b_{2}, \cdots, b_{p}\right) \in \Gamma_{p}$ such that $\beta_{i}=$ $\sigma_{p-i}(b)\left(\begin{array}{c}n \\ k-p+i\end{array}\right), i=0,1, \cdots p$

(3) For $p \geq k \geq n$,

$$
c \chi^{n}=\beta_{0} \chi^{n}+\beta_{1} \chi^{n-1} \wedge \omega+\cdots+\beta_{n} \omega^{n}, c=\sum_{i=0}^{n} \beta_{i} c_{i},
$$

for which we require the existence of $a b=\left(b_{1}, b_{2}, \cdots, b_{p}\right) \in \Gamma_{p}$ such that $\beta_{i}=$ $\sigma_{k-i}(b)\left(\begin{array}{c}n \\ i\end{array}\right), i=0,1, \cdots, n ;$

(4) For $k>p$ and $k \geq n$,

$$
c \chi^{n}=\beta_{0} \chi^{n+p-k} \wedge \omega^{k-p}+\beta_{1} \chi^{n+p-k-1} \wedge \omega^{k-p+1}+\cdots+\beta_{n+p-k} \omega^{n}, c=\sum_{i=0}^{n+p-k} \beta_{i} c_{k-p+i}
$$

where we require there exist some $b=\left(b_{1}, b_{2}, \cdots, b_{p}\right) \in \Gamma_{p}$ such that $\beta_{i}=\sigma_{p-i}(b)\left(\begin{array}{c}n \\ k-p+i\end{array}\right), i=$ $0,1, \cdots, n+p-k$. 
Remark 5.3. It is due to our specific method that $\beta_{i}$ 's have certain combinatorial constraints. We expect to remove these technical constraints in future works.

We finish the discussion with a geometric application.

Consider $[\chi]=[\omega]+\epsilon[a]$, where $[a] \in H^{1,1}(M)$ and $\epsilon \in \mathbb{R}$. Since $\omega$ is in the cone $\mathcal{C}_{k}$, and the cone is obvious open, then for $|\epsilon|$ small, $[\chi] \in \mathcal{C}_{k}$ for any $k \in\{1, \cdots, n\}$. Thus, by Theorem 1.1, we have $\chi \in[\chi]$ such that

$$
\frac{\chi^{n-k} \wedge \omega^{k}}{\chi^{n}}=c_{k}
$$

On the other hand, it is easy to check that, on the manifold $M$, we have the following point-wise inequalities:

$$
\frac{\chi^{n-1} \wedge \omega}{\chi^{n}} \geq \frac{\chi^{n-2} \wedge \omega^{2}}{\chi^{n-1} \wedge \omega} \geq \cdots \geq \frac{\chi^{n-k} \wedge \omega^{k}}{\chi^{n-k+1} \wedge \omega^{k-1}}
$$

where any equality holds iff $\chi=\lambda \omega$ for some constant $\lambda$. Thus,

$$
\frac{\chi^{n-1} \wedge \omega}{\chi^{n}} \geq\left[\frac{\chi^{n-1} \wedge \omega}{\chi^{n}} \cdot \frac{\chi^{n-2} \wedge \omega^{2}}{\chi^{n-1} \wedge \omega^{1}} \cdots \cdot \frac{\chi^{n-k} \wedge \omega^{k}}{\chi^{n-k+1} \wedge \omega^{k-1}}\right]^{\frac{1}{k}}=\left(c_{k}\right)^{\frac{1}{k}} .
$$

This leads to

$$
\frac{\int_{M} \chi^{n-1} \wedge \omega}{\int_{M} \chi^{n}} \geq\left(c_{k}\right)^{\frac{1}{k}}=\left[\frac{\int_{M} \chi^{n-k} \wedge \omega^{k}}{\int_{M} \chi^{n}}\right]^{\frac{1}{k}} .
$$

Notice that (5.12) is independent of the choice of $\chi \in[\chi]$. Notice $[\chi]=[\omega]+\epsilon[a]$. Take $k=2$, and expand both sides of (5.12) as a series of $\epsilon$, then let $\epsilon \rightarrow 0$, we get the following inequality:

$$
\left(\int_{M} \omega^{n-2} \wedge a^{2}\right)\left(\int_{M} \omega^{n}\right) \leq \frac{n-1}{n(n-2)}\left(\int_{M} \omega^{n-1} \wedge a\right)^{2},
$$

where the identity holds iff $[a]=\lambda^{\prime}[\omega]$ for some constant $\lambda^{\prime}$. This is exactly the RiemannHodge bi-linear relation for $(1,1)$-classes (see, e.g., [GH]).

\section{APPENDIX A}

In this appendix, we first present another proof of Remark 2.3. For the convenience of readers, we restate it as the following:

Proposition A.1. Let $g=\sigma_{k}^{\frac{1}{k}}(\chi)$, and $\chi \in \Gamma_{n}$. Let $g_{i}:=\frac{\partial g}{\partial \chi_{i}}, g_{i j}:=\frac{\partial^{2} g}{\partial \chi_{i} \partial \chi_{j}}$. Then the matrix $g_{i j}+\frac{g_{i}}{\chi_{j}} \delta_{i j}$ is nonnegative.

Proof. Step 1.

Consider $h:=\sigma_{k}\left(\chi^{\frac{1}{k}}\right)$. Use the same notation as above, we claim

$$
h_{i j}+\frac{h_{i}}{\chi_{j}} \delta_{i j} \geq 0 .
$$


Direct computation shows that:

$$
\begin{aligned}
h_{i} & =\frac{1}{k} \sigma_{k-1}\left(\chi^{\frac{1}{k}} \mid i\right) \chi_{i}^{\frac{1}{k}-1} \\
h_{i j} & =\frac{1}{k^{2}} \sigma_{k-2}\left(\chi^{\frac{1}{k}} \mid i, j\right) \chi_{i}^{\frac{1}{k}-1} \chi_{j}^{\frac{1}{k}-1}+\frac{1}{k}\left(\frac{1}{k}-1\right) \sigma_{k-1}\left(\chi^{\frac{1}{k}} \mid i\right) \chi_{j}^{\frac{1}{k}-2} \delta_{i j} .
\end{aligned}
$$

Introduce the following notation: for $I=\left(i_{1}, i_{2}, \cdots i_{l}\right)$ an arbitrary index set of length $l$, let $\sigma_{k ; I}:=\sum_{|I|=k} \chi_{I} \sigma_{k-l}(\chi \mid I)$, where $\chi_{I}=\chi_{i_{1}} \chi_{i_{2}} \cdots \chi_{i_{l}}$. Basically, it is the collection of terms in which indices $i \in I$ appear. In this notation, we can rewrite (A.1), (A.2) as:

$$
\begin{aligned}
h_{i} & =\frac{\sigma_{k ; i}}{k \chi_{i}}, \\
h_{i j} & =\frac{\sigma_{k ; i, j}}{k^{2} \chi_{i} \chi_{i}}, \quad \text { for } i \neq j, \\
h_{i i} & =\frac{1}{k}\left(\frac{1}{k}-1\right) \frac{\sigma_{k ; i}}{\chi_{i}^{2}} .
\end{aligned}
$$

So $h_{i j}+\frac{h_{i}}{\chi_{j}} \delta_{i j}$ equals:

$$
\left[\begin{array}{ccccc}
\frac{\sigma_{k ; 1}}{k^{2} \chi_{1}^{2}} & \frac{\sigma_{k ; 1,2}}{k^{2} \chi_{1} \chi_{2}} & \cdot & \cdot & \frac{\sigma_{k ; 1, n}}{k^{2} \chi_{1} \chi_{n}} \\
\frac{\sigma_{k ; 1,2}}{k^{2} \chi_{1} \chi_{2}} & \frac{\sigma_{k ; 2}}{k^{2} \chi_{2}^{2}} & & & \\
\cdot & & \cdot & \\
\cdot & & & \cdot & \\
\frac{\sigma_{k ; 1, n}}{k^{2} \chi_{1} \chi_{n}} & \cdot & & & \frac{\sigma_{k ; n}}{k^{2} \chi_{n}^{2}}
\end{array}\right]
$$

Then it is equivalent to show that

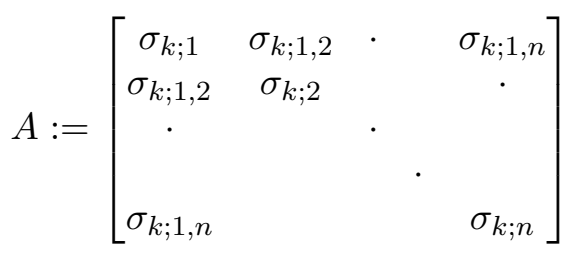

is nonnegative. For an index set $I$, Let $E_{I}$ be the matrix having entry 1 in $i$-th row and $j$-th column of an $n \times n$ matrix, where $i, j \in I$, and entry 0 elsewhere. It is clear that $E_{I}$ is nonnegative. Moreover, we have the following nice decomposition:

$$
A=\sum_{|I|=k} \chi_{I} E_{I} \geq 0
$$

Thus

Step 2.

$$
h_{i j}+\frac{h_{i}}{\chi_{j}} \delta_{i j} \geq 0
$$

We claim

$$
h_{i j}+\frac{h_{i}}{\chi_{j}} \delta_{i j}-\frac{h_{i} h_{j}}{h} \geq 0 .
$$

We use a nice trick due to Andrews [A]. Since $h$ is homogenous of degree $1, h_{i} \chi_{i}=h$. Differentiate both sides, one gets $h_{i j} \chi_{i}=0$. Consequently,

$$
\left(h_{i j}+\frac{h_{i}}{\chi_{j}} \delta_{i j}-\frac{h_{i} h_{j}}{h}\right) \chi_{i} \chi_{j}=0
$$


i.e. $\chi$ is a null vector. In order to show $h_{i j}+\frac{h_{i}}{\chi_{j}} \delta_{i j}-\frac{h_{i} h_{j}}{h} \geq 0$, one then only need to look at a subspace transversal to the null vector $\chi=\left(\chi_{1}, \cdots, \chi_{n}\right)$. Naturally, we choose the subspace defined by $\left\{\xi \mid h_{i} \xi_{i}=0\right\}$. Then $\left(h_{i j}+\frac{h_{i}}{\chi_{j}} \delta_{i j}-\frac{h_{i} h_{j}}{h}\right) \xi_{i} \xi_{j}=\left(h_{i j}+\frac{h_{i}}{\chi_{j}} \delta_{i j}\right) \xi_{i} \xi_{j}$, which is nonnegative from step 1 .

Step 3. $g\left(\chi_{1}, \cdots \chi_{n}\right)=h^{\frac{1}{k}}\left(\chi_{1}^{k}, \cdots, \chi_{n}^{k}\right)$, a simple computation shows that:

$$
g_{i j}+\frac{g_{i}}{\chi_{j}} \delta_{i j}=k h^{\frac{1}{k}-1}(\lambda) \lambda_{i}^{1-\frac{1}{k}} \lambda_{j}^{1-\frac{1}{k}}\left[h_{i j}+\frac{h_{i}}{\lambda_{j}} \delta_{i j}-\frac{k-1}{k} \frac{h_{i} h_{i}}{h}\right]
$$

where $\chi_{i}^{k}=\lambda_{i}$. Thus,

$$
h_{i j}+\frac{h_{i}}{\lambda_{j}} \delta_{i j}-\frac{k-1}{k} \frac{h_{i} h_{i}}{h} \geq h_{i j}+\frac{h_{i}}{\lambda_{j}} \delta_{i j}-\frac{h_{i} h_{i}}{h} \geq 0,
$$

the last inequality is due to step 2 . The proof is thus completed.

Remark A.2. It is clear from the above proof that the conclusion of Proposition holds for $g=\sigma_{k}^{\epsilon}(\chi)$, with $\epsilon>0$.

\section{APPENDIX B}

In this appendix, we summarize the classical parabolic Krylov-Evans theory that are applied in this paper. In particular, we deduce time $C^{\frac{\alpha}{2}}$ estimates for $\partial \bar{\partial} \varphi$ for (1.4). These estimates are local in nature. This proof is essentially due to Lihe Wang Wang2.

In the parabolic case, it is also convenient to introduce the following regularity notation. We say $\varphi=\varphi(x, t) \in C^{2, \alpha}$ in the parabolic sense if and only if $\varphi \in C^{2, \alpha}$ in spatial variable $x \in \mathbb{R}^{n}$, and $\varphi \in C^{1, \frac{\alpha}{2}}$ in time variable $t \in \mathbb{R}$ in the usual sense. Different regularity

is due to different scaling of spatial and time variables. We will also write $C_{x, t}^{2+\alpha, 1+\frac{\alpha}{2}}$ to indicate regularity respectively. For a thorough exposition, we refer readers to Wang1 and Wang2.

The fundamental tool to attack nonlinear parabolic equation is following:

Theorem B.1 (Krylov-Safanov). Let $\varphi$ be a solution of

$$
\varphi_{t}=a_{i j}(x, t) \varphi_{i j}
$$

in $\mathbf{Q}_{1}$, and $a_{i j}$ is uniform elliptic, then $\varphi$ is in $C_{l o c}^{\alpha}\left(\mathbf{Q}_{1}\right)$, i.e., $\varphi$ is $C^{\alpha}$ in spatial and $\varphi$ is $C^{\frac{\alpha}{2}}$ in time.

The parabolic equation we have is:

$$
\frac{\partial \varphi}{\partial t}=c+F(\partial \bar{\partial} \varphi)
$$


By Theorem 3.1, $F$ is a uniform elliptic, concave operator. Taking derivative with respect to $t$ both sides of $(\underline{\mathrm{B} .2})$, one has

$$
\varphi_{t t}=F^{i \bar{j}}\left(\varphi_{t}\right)_{i \bar{j}}
$$

By Theorem B1, $\varphi_{t}$ is $C_{x}^{\alpha}$. Thus $(\overline{\mathrm{B} .2})$ can be viewed as an elliptic equation. Then by the elliptic Krylov-Evans theory, one has spatial $C^{\alpha}$ estimate on $D_{x}^{2} \varphi$. To have $C^{\alpha}$ estimate for $D_{x}^{2} \varphi$, it is sufficient to show time $C^{\frac{\alpha}{2}}$ estimate. Since the problem is local in nature, we just need to prove time $C^{\frac{\alpha}{2}}$ estimate at $(0,0)$.

Since $\varphi$ is $C^{2, \alpha}$ in spatial, there exist two quadratic polynomials $P_{t}(x)$ and $P_{0}(x)$ such that

$$
\begin{aligned}
& \left|\varphi(x, t)-P_{t}\right| \leq C|x|^{2+\alpha}, \quad|x| \leq \sqrt{t} \\
& \left|\varphi(x, 0)-P_{0}\right| \leq C|x|^{2+\alpha}, \quad|x| \leq \sqrt{t}
\end{aligned}
$$

Also, since $\varphi_{t} \in C^{\alpha}$

$$
\begin{gathered}
\left|\varphi(x, t)-\varphi(x, 0)-t \varphi_{t}(x, 0)\right| \leq C t^{1+\frac{\alpha}{2}}, \quad|x| \leq \sqrt{t} . \\
\left|\varphi_{t}(x, 0)-\varphi_{t}(0,0)\right| \leq C|x|^{\alpha} .
\end{gathered}
$$

By (B.4),(B.5) and (B.6) together, we have

$$
\left|P_{t}(x)-P_{0}(x)-t \varphi_{t}(x, 0)\right| \leq C t^{1+\frac{\alpha}{2}} .
$$

(B.8) and (B.7) imply that

$$
\left|P_{t}(x)-P_{0}(x)\right| \leq C t^{1+\frac{\alpha}{2}}, \quad|x| \leq \sqrt{t} .
$$

For a quadratic polynomial, one has

$$
\left\|D_{x}^{2} P\right\|_{B_{r}} \leq C \frac{\|P\|_{L^{\infty}\left(B_{r}\right)}}{r^{2}}
$$

Therefore,

$$
\left\|D_{x}^{2} P_{t}-D_{x}^{2} P_{0}\right\|_{B_{\sqrt{t}}} \leq C \frac{\left\|P_{t}-P_{0}\right\|_{L^{\infty}\left(B_{\sqrt{t}}\right)}}{t} \leq C t^{\frac{\alpha}{2}}
$$

which implies that

$$
\left\|D_{x}^{2} \varphi(0, t)-D_{x}^{2} \varphi(0,0)\right\| \leq C t^{\frac{\alpha}{2}}
$$

\section{REFERENCES}

[A] B. Andrews, Pinching estimates and motion of hypersurfaces by curvature functions, J. Reine Angew. Math. 608 (2007), 17-33.

[C] H. Cao, Deformation of Kähler metrics to Kähler-Einstein metrics on compact Kähler manifolds, Invet. Math. (1985), 81,359-372.

[Ch1] X.Chen, On the lower bound of the Mabuchi energy and its application. Internat. Math. Res. Notices (2000), no. 12, 607-623.

[Ch2] X.Chen, A new parabolic flow in Kähler manifolds. Comm. Anal. Geom. 12 (2004), no. 4, 837-852.

[ChT] X.Chen, G.Tian, Ricci flow on Kähler-Einstein surfaces. Invent. Math. 147 (2002), no. 3, 487-544.

[D] S.Donaldson Moment maps and diffeomorphisms, Asian J. Math. 3, no. 1 (1999), 1-16.

[GH] P.Griffiths, J.Harris, Principles of algebraic geometry. Reprint of the 1978 original. John Wiley \& Sons, Inc., New York, 1994. xiv+813 pp. ISBN: 0-471-05059-8. 
[GM] P. Guan, X. Ma, The Christoffel-Minkowski problem I: convexity of solutions of a Hessian equation Inventiones Mathematicae, V.151 (2003), 553-577.

[GLZ] P. Guan, Q. Li, X. Zhang, A uniqueness theorem in Kähler geometry, Math.Ann. 345(2009), no.2, 377-393.

[K] Krylov, N. V. Nonlinear elliptic and parabolic equations of the second order. Translated from the Russian by P. L. Buzytsky [P. L. Buzytski]. Mathematics and its Applications (Soviet Series), 7. D. Reidel Publishing Co., Dordrecht, 1987. xiv+462 pp. ISBN: 90-277-2289-7.

[L] G. M. Lieberman, Second order parabolic differential equations. World Scientific Publishing Co., Inc., River Edge, NJ, 1996. xii+439 pp. ISBN: 981-02-2883-X.

[S] J.Spruck, Geometric aspects of the theory of fully nonlinear elliptic equations, http://www.math.jhu.edu/ js/msri.notes.pdf

[SW] J.Song, B.Weinkove, On the convergence and singularities of the $J$-flow with applications to the Mabuchi energy, Comm. Pure Appl. Math. 61 (2008), no. 2, 210-229.

[T1] G.Tian, On Kähler-Einstein metrics on certain Kähler mainfolds with $C_{1}(M)>0$, Invent. Math. 89 (1987), no.2, 225-246.

[T2] G.Tian, Bott-Chern forms and geometric stability, Discrete Contin. Dynam. Systems 6 (2000), no. 1, $211-220$.

[Wang1] L. Wang, On the regularity theory of fully nonlinear parabolic equations. I. Comm. Pure Appl. Math. 45 (1992), no. 1, 27-76.

[Wang2] L. Wang, On the regularity theory of fully nonlinear parabolic equations. II. Comm. Pure Appl. Math. 45 (1992), no. 2, 141-178.

[W1] B.Weinkove, Convergence of the J-flow on Kähler surfaces, Comm. Anal. Geom. 12 (2004), no. 4, 949-965.

[W2] B.Weinkove, On the $J$-flow in higher dimensions and the lower boundedness of the Mabuchi energy. J. Differential Geom. 73 (2006), no. 2, 351-358.

[Y] S.Yau On the Ricci curvature of a compact Kähler manifold and the complex Monge-Ampère equation, I. Comm. Pure Appl. Math. 31 (1978), no.3, 339-411.

Department of Mathematics, University of Iowa, Iowa City, IA 52245

E-mail address: haofang@math.uiowa.edu

Department of Mathematics, University of Iowa, Iowa City, IA 52245

E-mail address: mijlai@math.uiowa.edu

Department of Mathematics, University of Science and Technology of China, Hefei, 230026, Anhui Province, CHINA

E-mail address: xinan@ustc.edu.cn 